\title{
Flexible Control of Safety Margins for Action Based on Environmental Variability
}

\author{
Alkis M. Hadjiosif ${ }^{1}$ and Maurice A. Smith ${ }^{1,2}$ \\ ${ }^{1}$ School of Engineering and Applied Sciences, and ${ }^{2}$ Center for Brain Science, Harvard University, Cambridge, Massachusetts 02138
}

To reduce the risk of slip, grip force (GF) control includes a safety margin above the force level ordinarily sufficient for the expected load force (LF) dynamics. The current view is that this safety margin is based on the expected LF dynamics, amounting to a static safety factor like that often used in engineering design. More efficient control could be achieved, however, if the motor system reduces the safety margin when LF variability is low and increases it when this variability is high. Here we show that this is indeed the case by demonstrating that the human motor system sizes the GF safety margin in proportion to an internal estimate of LF variability to maintain a fixed statistical confidence against slip. In contrast to current models of GF control that neglect the variability of LF dynamics, we demonstrate that GF is threefold more sensitive to the SD than the expected value of LF dynamics, in line with the maintenance of a 3-sigma confidence level. We then show that a computational model of GF control that includes a variability-driven safety margin predicts highly asymmetric GF adaptation between increases versus decreases in load. We find clear experimental evidence for this asymmetry and show that it explains previously reported differences in how rapidly GFs and manipulatory forces adapt. This model further predicts bizarre nonmonotonic shapes for GF learning curves, which are faithfully borne out in our experimental data. Our findings establish a new role for environmental variability in the control of action.

Key words: force field; grip force; internal model; motor learning; safety margin; variability

\section{Introduction}

A skilled cellist masterfully controls the dynamics of his bow via a finely tuned pattern of motor output that ensures that the bow slides at the right speed, touching the right string, at the right time, with the right amount of force. Yet as Pablo Casals, the preeminent cellist of the early $20^{\text {th }}$ century, learned all too well, this remarkable control fails completely if one loses grip on the bow, even momentarily. In his highly anticipated 1907 Vienna debut, a stage-frightened Casals lost his grip, sending his bow flying into the audience. The accommodating Viennese concertgoers quickly returned the bow, and Casals went on to deliver a landmark performance. Yet, among thousands of flawlessly executed movements, that single bow slip was what he remembered 50 years later (Goldsmith, 1956).

Casal's story invites us to consider the importance of robust grip force (GF) control that generally prevents accidental slips. Indeed, how does the brain avoid making them all the time? To prevent such accidents, GFs generally include a safety margin

\footnotetext{
Received May 9, 2014; revised April 2, 2015; accepted May 5, 2015.

Author contributions: A.M.H. and M.A.S. designed research; A.M.H. performed research; A.M.H. and M.A.S. analyzed data; A.M.H. and M.A.S. wrote the paper.

This work was supported in part by the McKnight Scholar Award, a Sloan Research Fellowship, and a grant from the National Institute on Aging (R01 AG041878) to M.A.S. We thank Simon Orozco and Emerson Fang for their help with the experiments, Jordan Brayanov and Joel Greenwood for their help with instrumentation, and Jasmine Bailey, Andrew Brennan, Jordan Brayanov, and Yohsuke Miyamoto for helpful discussions.

The authors declare no competing financial interests.

Correspondence should be addressed to Maurice A. Smith, Harvard University, School of Engineering and Applied Sciences, 29 0xford Street, Cambridge, MA 02138.E-mail: mas@seas.harvard.edu.

DOI:10.1523/JNEUROSCI.1883-14.2015

Copyright $\odot 2015$ the authors $\quad 0270-6474 / 15 / 359106-16 \$ 15.00 / 0$
}

above the force level usually sufficient to maintain grasp, reducing the chance that unexpected perturbations, misestimation of load forces (LFs), or errors in GF production result in slippage (Westling and Johansson, 1984).

Previous work has suggested that the predictive control of GF acts to maintain a constant GF/LF ratio, which, in line with the maintenance of a safety margin, is consistently higher than the so-called slip ratio, the minimum GF/LF ratio that avoids slip (Johansson and Westling, 1984, 1988; Cole and Johansson, 1993). Moreover, previous work has generally maintained that both the overall GF/LF ratio and the ratio margin [(GF/LF) minus slip ratio] are proportional to the slip ratio (Westling and Johansson, 1984), making the GF safety margin proportional to the expected slip force. Thus the current theory corresponds to a margin described by a fixed safety factor, like that widely used in engineering design (ACI Committee 318, 2005; NASA, 2008). For example, with a $20 \%$ safety factor, a bridge built for a 1000 ton load would be designed to withstand 1200 tons, or when holding a $5 \mathrm{~kg}$ object, one would apply a GF sufficient for $6 \mathrm{~kg}$.

We hypothesize that the CNS does not maintain a fixed safety factor for GF control, but instead adaptively controls the safety margin based on on-line estimates of the uncertainty about environmental dynamics. This allows the motor system to efficiently balance effort against the risk of slip. We can afford to keep the safety margin small without risking slip when environmental variability is low, but would benefit from maintaining a large safety margin to prevent frequent slips when variability is highanalogous to the notion that, when driving, one should keep a larger distance from erratically behaving vehicles. Quantitatively, a variability-based safety margin could provide a fixed statistical 
A

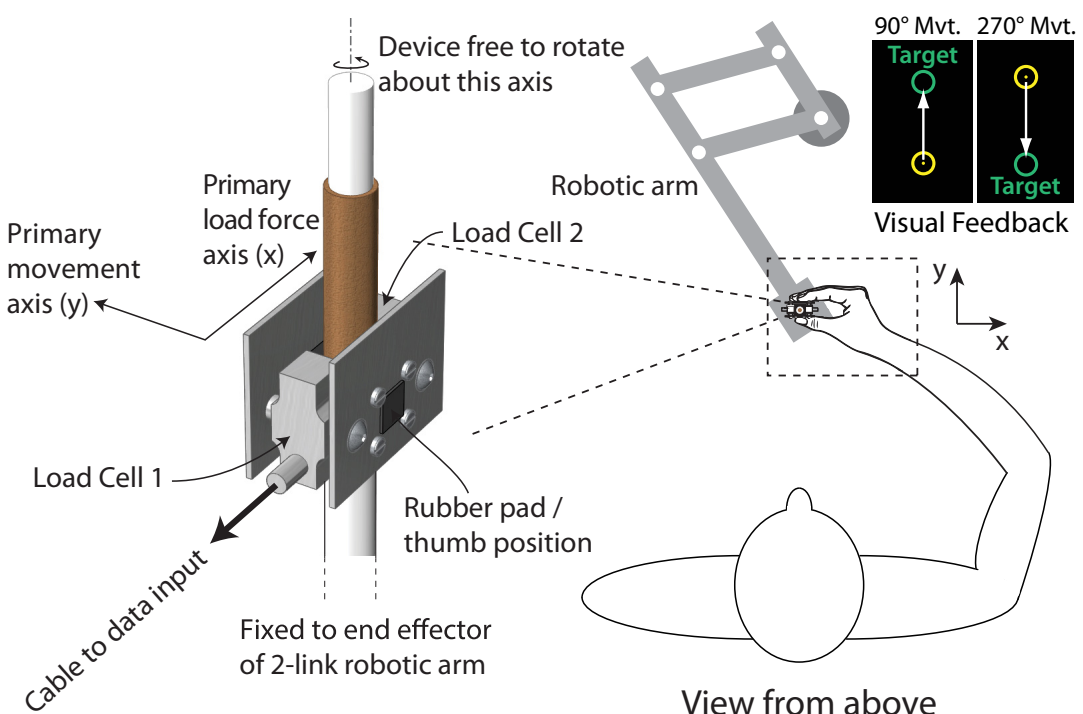

B
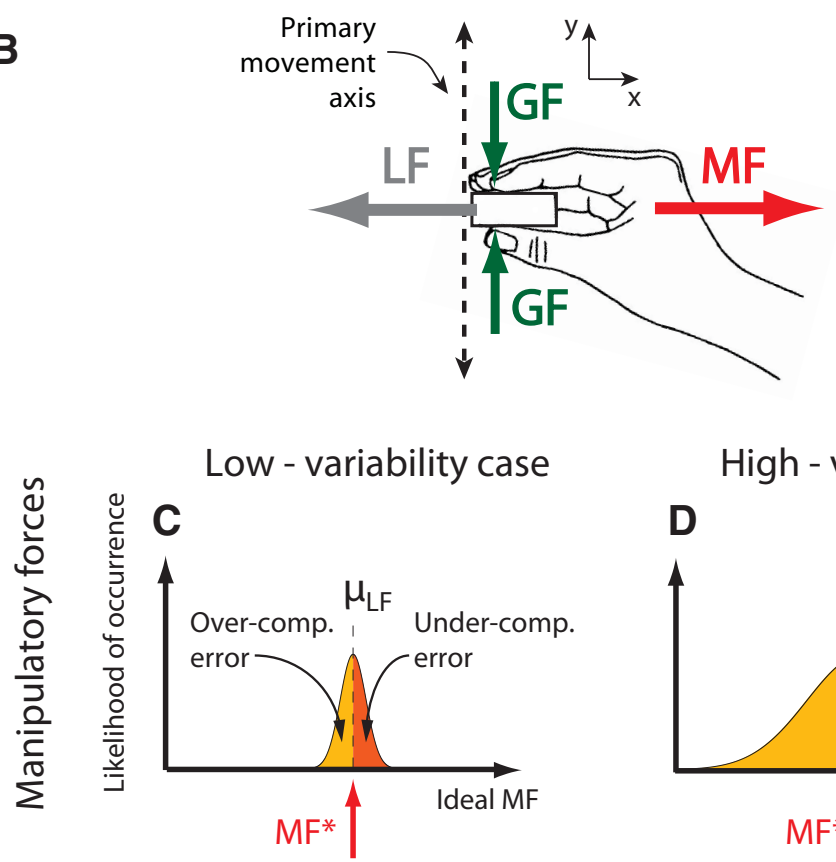

High - variability case
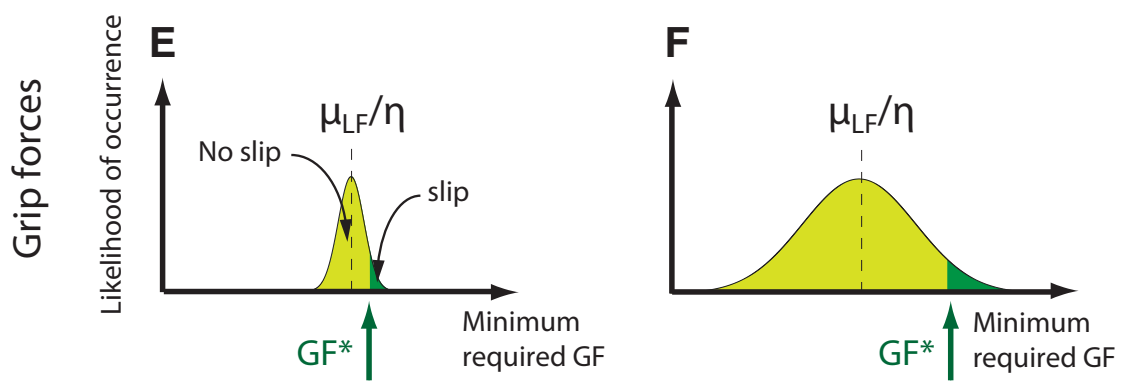

Figure 1. GFs and MFs in variable environments. $A$, Diagram of the grasping device (left) and top view of the experiment setup. $\boldsymbol{B}$, Top view of the grasped object illustrating the forces exerted on and by the subject during movement. Rightward MFs can compensate leftward LFs and compressive GFs maintain grasp. C, D, MF compensation in a low-variability (C) or a high-variability (D) environment, both with the same expected value $\mu_{\mathrm{LF}}$. The optimal MF level is equal to the expected value of the perturbation, which minimizes the amount of overcompensation and undercompensation in both cases. $\boldsymbol{E}, \boldsymbol{F}, \mathrm{GF}$ control in the same environments. The GF required to avoid slip has a mean value of the mean load divided by the coefficient of friction $\left(\mu_{\mathrm{LF}} / \eta\right)$ in both cases. However, note that, in $50 \%$ of the cases, using this expected value (vertical dashed line) would result in slip whenever the required GF exceeded this value. In a low-variability environment $(\boldsymbol{E})$, using a $\mathrm{GF}\left(\mathrm{GF}^{*}\right)$ that incorporates a small safety margin above this expected value ensures that the chance of slippage is low (dark green area under the bell curve). However, in a high-variability environment $(\boldsymbol{F})$, a larger safety margin would be required to maintain a low slip probability. This leads to the hypothesis that GF control includes a safety margin that scales with environmental variability. confidence level against failure, removing the dependence on environmental variability.

Altogether, we propose that GF control is based on an additive combination of what is directly known about the environment (its expected dynamics) to estimate the ordinarily sufficient action, and the known unknown (the environment's variability) to size a safety margin based on statistical confidence. Because a safety margin of 2-3 SDs would be required to maintain 95-99\% confidence bounds (Wasserman, 2004), this hypothesis would predict GF to be more sensitive to the SD of the environment than to its mean, a radical departure from current thinking in which environmental variability has no role (Johansson and Westling, 1984, 1988; Cole and Johansson, 1993; Flanagan and Wing, 1993, 1997; Flanagan et al., 2003).

In contrast to GF control, safety margins do not readily apply to manipulatory force (MF) control, because both excessive and inadequate forces result in motor errors. Accordingly, MF control is largely based on estimates of the expected value of dynamics (Scheidt et al., 2001; Takahashi et al., 2001; Mawase and Karniel, 2012) without a safety margin. We thus designed a series of experiments to compare how the control of GFs and MFs is affected by environmental variability.

\section{Materials and Methods}

Participants and ethics statement

A total of 70 healthy individuals without known neurological disease (21 male, age $22.1 \pm 4.4$ years) participated in the two experiments. All subjects were naive to the purpose of the experiments and provided informed consent. Two subjects were (self-reportedly) left handed, but used their right arm for the experiment, as the task did not require using the dominant hand. All experiment protocols were approved by the Harvard University Committee on the Use of Human Subjects in Research.

\section{General task}

Participants grasped a custom-designed GF measurement device using a pinch grip (Fig. $1 A)$. This object consisted of two load cells (LRF300, Futek Advanced Sensor Technology) connecting two rubber-coated parallel grip surfaces $28.3 \mathrm{~mm}$ apart. We restricted subjects' choice of grasp position to avoid graspconfiguration-dependent differences in GF adaptation (Fu et al., 2010) that might obscure effects related to differences in experienced dynamics. This device was attached to a robotic manipulandum (Interactive Motion Technologies) in such a way that it could freely rotate about the vertical axis, allowing an orientation lateral to movement direction to be maintained throughout each movement (Fig. 1A). 
While grasping the object, subjects performed $10 \mathrm{~cm}$ reaching arm movements toward or away from the chest aimed at $1 \mathrm{~cm}$ circular targets displayed on a vertically oriented LCD screen in front of them. During each movement, the subject's hand position was represented by a 3-mmdiameter cursor. Subjects were trained to strive for a movement duration of $500 \pm 50 \mathrm{~ms}$, based on visual feedback about movement speed following each trial. Subjects were instructed to move directly to each target, and to maintain a fixed grasp orientation as illustrated in Figure $1 \mathrm{~A}$. After a short familiarization session, a leftward LF consisting of static and dynamic components was applied to the object according to the following equations (Eq. 1):

$$
\left[\begin{array}{l}
F_{x} \\
F_{y}
\end{array}\right]=\left[\begin{array}{c}
F_{0} \\
0
\end{array}\right]+\left[\begin{array}{cc}
0 & b \\
-b & 0
\end{array}\right]\left[\begin{array}{l}
v_{x} \\
v_{y}
\end{array}\right]
$$

where $F_{0}$ is the static offset and $b$ is the strength of a viscous force field (FF), which could change from one trial to the next. In Equation 1, $y$ is the axis tangential to the movement direction, with positive $y$ away from the body, whereas $x$ is lateral to it with positive $x$ toward the right. We made the static force offset $F_{0}$ large enough to ensure that the overall LF would always remain leftward, even for negative values of $b$ (Fig. 2C). Experiments consisted of baseline and training sessions, with breaks every $6-7$ min. Each trial consisted of two movements, one toward $\left(270^{\circ}\right.$ direction) and one away from the chest $\left(90^{\circ}\right.$ direction). Note that, throughout this manuscript, we refer to "positive $b$ " as one that increases the magnitude of the exerted LF regardless of the movement direction (i.e., a "positive $b$ " would refer to $b>0$ for $270^{\circ}$ movements and to $b<0$ for $90^{\circ}$ movements). Thus, the sign of $b$ in Equation 1 is correct when $270^{\circ}$ movements are considered, whereas for $90^{\circ}$ movements this sign has to be inverted.

Our experiments consisted of three main conditions (Exps. 1a, 2a, and 2b) and three controls (Exps. 1b, 1c, and 2c). These data were obtained from five groups of participants, which did not overlap in membership. One group performed both Experiments $1 \mathrm{c}$ and $2 \mathrm{c}$, and the other four participant groups each performed single experiments (1a, 1b, 2a, and $2 b$, respectively).

\section{Experiments $1 a, 1 b$, and $1 c$}

Experiments $1 \mathrm{a}, 1 \mathrm{~b}$, and $1 \mathrm{c}$ were designed to investigate the effect of environmental variability on GF and MF control. In Experiment $1 \mathrm{a}(\mathrm{N}=$ 11 ), the force offset $F_{0}$ was held constant at $4 \mathrm{~N}$, while the viscous FF coefficient $b$ was varied from trial to trial; $b$ was drawn from a Gaussian distribution with mean zero and SD $\sigma_{b}$, which depended on the type of experiment block: zero-variability, low-variability, medium-variability, and high-variability blocks corresponding to $\sigma_{b}=0,1.8,3.6$, or $5.4 \mathrm{Ns} / \mathrm{m}$ (Fig. $2 A, B$ ). During the baseline period, $b$ was set to zero (i.e., only the offset force was experienced). After the baseline period, participants were exposed to 18 50-trial blocks. The first and last were zero-variability blocks and the middle 16 randomly alternated between the zerovariability, low-variability, medium-variability, and high-variability conditions such that each was presented four times. Brief $\sim 1$ min rest breaks were instituted every 50 trials and occurred 10 trials before the onset of each block. Experiment $1 \mathrm{~b}(\mathrm{~N}=23)$ was similar to Experiment la except that $F_{0}=3 \mathrm{~N}, \sigma_{b}=0,1.2,2.4$, or $3.6 \mathrm{Ns} / \mathrm{m}$, blocks were 100 trials in duration, and there were a total of eight training blocks, all randomly ordered, such that each variability condition was presented twice. Because the blocks were longer, breaks were given both 10 trials before and 40 trials after the onset of each block, to maintain 50-trial intervals between breaks. Experiment $1 \mathrm{c}(N=17)$ was similar to Experiment la except that $b$ was drawn from a Gaussian distribution with a mean that was $+3.75 \mathrm{Ns} / \mathrm{m}$, and a 240 -trial constant $+3.75 \mathrm{Ns} / \mathrm{m} \mathrm{FF}$ epoch (Exp. 2c; see below) was inserted between the 18-block experiment period and baseline.

Experiments $2 a, 2 b$, and $2 c$

Experiments $2 \mathrm{a}, 2 \mathrm{~b}$, and $2 \mathrm{c}$ were designed to investigate how a sudden change in the environment would affect GF and MF control. For Experiments $2 \mathrm{a}$ and $2 \mathrm{~b}$, the force offset $F_{0}$ was held constant at $3 \mathrm{~N}$. Following the 80 -trial baseline period, $b$ was switched from zero to a value of \pm 7.5 $\mathrm{Nm} / \mathrm{s}$ at the onset of the 240-trial training period. In Experiment $2 \mathrm{a}(\mathrm{N}=$ 18) participants trained with leftward FFs that we deem positive ( $+F F)$ because they increase the LF relative to $F_{0}(b=+7.5 \mathrm{Ns} / \mathrm{m}$ and -7.5 $\mathrm{Ns} / \mathrm{m}$ for $270^{\circ}$ and $90^{\circ}$ movement directions, respectively), whereas in Experiment $2 \mathrm{~b}(N=18)$ participants trained with rightward FFs that we deem negative $(-\mathrm{FF})$ because they decrease the LF relative to $F_{0}$. We inserted short rest breaks every 64 trials, and the training period began 16 trials after a break to dissociate any effect of the break from the onset of the FF. Experiment 2c $(N=17)$ was similar to Experiment 2a except that it was immediately followed by another experiment (1c), the force offset $F_{0}$ was held constant at $4 \mathrm{~N}$, and the FF strength was $b=+3.75 \mathrm{Ns} / \mathrm{m}$ instead of $+7.5 \mathrm{Ns} / \mathrm{m}$.

\section{Data analysis and computational modeling}

Data inclusion criteria. Trials in which the peak velocity was $<0.2 \mathrm{~m} / \mathrm{s}$ or $>0.5 \mathrm{~m} / \mathrm{s}(<0.4 \%$ of trials in total) were excluded. In Experiment 2, we found strong GF increases in the first few trials following each rest break. To remove this postbreak artifact, we omitted the first four trials after every break from our main analyses for all experiments.

Estimation of adaptation levels for MFs and GFs. We measured MFs during error-clamp (EC) trials (Scheidt et al., 2000; Smith et al., 2006) interspersed with a frequency of 1 in 5 in a pattern that was random but which avoided consecutive EC trials. These trials minimize lateral error via the implementation of a virtual force channel with a high lateral stiffness of $6000 \mathrm{~N} / \mathrm{m}$ to isolate the feed-forward component of adaptation. We estimated the MF adaptation level on each EC trial by regressing (Smith et al., 2006; Joiner and Smith, 2008; Yousif and Diedrichsen, 2012) the baseline-subtracted (baseline defined as 250 to $100 \mathrm{~ms}$ before movement onset) force profile onto the velocity profile for that trial (on which the FF would have been based). The regression coefficient obtained is an estimate of the value of $b$ that the measured MF would have compensated for during each trial, because the viscous FFs we use scale the velocity profile by $b$ (Eq. 1 ).

We measured GFs during all trials using the measurement device described in the General task paragraph. The device consisted of two load cells sandwiched between the two grip surfaces (Fig. 1A). GFs were measured as the sum of the readings from these two load cells. We assessed the level of GF adaptation on each trial using the same method as for MFs: we regressed each baseline-subtracted GF profile onto the corresponding velocity profile for that trial and used the resulting regression coefficient as a measure of GF adaptation. To calculate the force adaptation level associated with each variability block, in Experiments 1a, 1b, and 1c, we averaged the regression coefficients obtained from Trial 16 to the end of the block. We omitted the first 15 trials to exclude potential aftereffects from the variability level during the preceding block. However, as we note in the Results section, changing the size of this selection window did not yield meaningful differences in results.

Estimation of the sensitivity of MFs and GFs to the SD and mean of LF dynamics. We estimated the sensitivity of GF and MF to the SD of environmental dynamics (i.e., the $\mathrm{GF} / \sigma_{\mathrm{LF}}$ and $\mathrm{MF} / \sigma_{\mathrm{LF}}$ sensitivities, respectively) in Experiment 1, where we measured GF and MF responses to different variability levels. Sensitivity to SD corresponded to the slope of Figure $3 A$ for GF and Figure $3 D$ for MF, as it was defined as the change in force level divided by the change in SD from Experiment 1, as shown in the following equation:

$$
\text { Sensitivity to standard deviation }=\frac{\Delta F}{\Delta \sigma_{\mathrm{LF}}}
$$

Correspondingly, we estimated the sensitivity of GF and MF to the mean of environmental dynamics (i.e., the $\mathrm{GF} / \mu_{\mathrm{LF}}$ and $\mathrm{MF} / \mu_{\mathrm{LF}}$ sensitivities, respectively) in Experiment 2, where we measured asymptotic GF and MF adaptation to one of three different step changes in the mean FF: 7.5 Ns/m (Exp. 2a), $-7.5 \mathrm{Ns} / \mathrm{m}$ (Exp. 2b), and $3.75 \mathrm{Ns} / \mathrm{m}$ (Exp. 2c). Sensitivity to the mean corresponded to the slope of Figure $3 B$ for GF and Figure $3 E$ for MF, as it was defined as change in GF between baseline and asymptote adaptation, divided by the corresponding change in the mean, as shown in the following equation:

$$
\text { Sensitivity to the mean }=\frac{\Delta F}{\Delta \mu_{\mathrm{LF}}}
$$


Computational model of combined mean-driven and variability-driven adaptation. Based on the results of Experiments 1a, 1b, 1c, 2a, 2b, and 2c presented in Figures 2 and 3, which show that GFs are sensitive to both the mean and variability of environmental dynamics, we developed a model to explain how GFs respond to a step change in environmental dynamics. This model posits GF control to be the sum of two components, one driven by an internal estimate of the expected LF and the other driven by an estimate of LF uncertainty. We refer to this as the additive combination of expected value and uncertainty (ACEVU) model. The model posits that the mean, $\mu$, and SD, $\sigma$, of the environmental dynamics (i.e., the variable $b$ ) at each trial $k$ are both estimated based on an exponential memory window with time constant $\tau$ (see Eq. 2 ). This is in line with recent work suggesting that the motor system registers a memory of squared errors that decreases exponentially from trial to trial (Landy et al., 2012); however, we note that any window type that weighs recent memories more heavily than older ones would yield qualitatively similar results. The computations required to generate the exponentially windowed estimates of $\mu$ and $\sigma$ required for the ACEVU model are shown in the following equation (Eq. 2):

$$
\begin{gathered}
\hat{\mu}(k+1)=\frac{\sum_{i=0}^{k} b(i) \cdot e^{-(k-i) / \tau}}{\sum_{i=0}^{k} e^{-(k-i) / \tau}}=\frac{\sum_{i=0}^{k} b(i) \cdot e^{i / \tau}}{\sum_{i=0}^{k} e^{i / \tau}} \\
=\left(\frac{1-e^{1 / \tau}}{1-e^{k / \tau}}\right) \sum_{i=0}^{k} b(i) \cdot e^{i / \tau}
\end{gathered}
$$$$
\hat{\sigma}^{2}(k+1)=\frac{\sum_{i=0}^{k}(b(i)-\hat{\mu}(i))^{2} \cdot e^{-(k-i) / \tau}}{\sum_{i=0}^{k} e^{-(k-i) / \tau}}
$$$$
=\frac{\sum_{i=0}^{k}(b(i)-\hat{\mu}(i))^{2} \cdot e^{i / \tau}}{\sum_{i=0}^{k} e^{i / \tau}}=\left(\frac{1-e^{1 / \tau}}{1-e^{k / \tau}}\right) \sum_{i=0}^{k}(b(i)-\hat{\mu}(i))^{2} \cdot e^{i / \tau}
$$

Note that Equation 2 can be rewritten recursively as follows (Eq. 3):

$$
\begin{gathered}
\hat{\mu}(k+1)=A \cdot \hat{\mu}(k)+B \cdot b(k) \\
\hat{\sigma}^{2}(k+1)=A \cdot \hat{\sigma}^{2}(k)+B \cdot(b(k)-\hat{\mu}(k))^{2} \\
A=\frac{1-e^{k / \tau}}{1-e^{(k+1) / \tau}}, \quad B=\left(1-e^{1 / \tau}\right) \frac{e^{k / \tau}}{1-e^{(k+1) / \tau}}
\end{gathered}
$$

The recursive formulation presented in Equation 3 illustrates an iterative implementation of mean and variability estimates that computes the effect of an exponential memory window without requiring the maintenance of separate memories for all of the individual movements in the memory window. Note that for $k$ sufficiently large (i.e., when the mean and variance have been estimated for a large number of trials compared with $\tau$ ), the coefficients $A$ and $B$ can be simplified and no longer depend on $k$, as shown in the following equation (Eq. 4):

$$
A=e^{-1 / \tau}, \quad B=1-e^{-1 / \tau}
$$

Estimating the mean-driven and variability-driven components of the adaptation curves. We began by calculating the shape of the force profiles $F(t)$ for the specific mean-driven and nonspecific variability-driven components of GF and MF adaptation by performing a common versus differential mode decomposition of the leftward and rightward FF force profiles, as shown in the following equation (Eq. 5):

$$
\begin{aligned}
& F_{C}=\frac{1}{2}\left(F_{+}+F_{-}\right) \\
& F_{D}=\frac{1}{2}\left(F_{+}-F_{-}\right)
\end{aligned}
$$

Such that $F_{+}=F_{C}+F_{D}$ and $F_{-}=F_{C}+F_{D}$ (Eq. 6). Here, the common mode $F_{C}$ (half of the sum) between the leftward and rightward FF force profiles ( $F_{+}$and $F_{-}$, respectively) isolates the nonspecific component of force profiles, whereas the differential mode $F_{D}$ (half of the difference) between these force profiles isolates the environment-specific component.

We then estimated the trial-to-trial adaptation curves for the perturbation-specific and perturbation-nonspecific components for each of the 36 subjects in Experiment $2 \mathrm{a} / 2 \mathrm{~b}$ by regressing GFs in each trial simultaneously onto the shapes of both the specific and nonspecific components (see Fig. $6 B, D$, gray curves).

Examining effects of EC-induced environmental variability. The external force patterns experienced during EC trials depend on the force applied by the subject and therefore do not exactly match the force applied during regular FF trials. Thus, having EC trials interspersed with the constant-value FF training trials in Experiment 2 would introduce additional variability in the effective FF, and this could result in increased variability-driven adaptation according to the ACEVU model. Thus, the increased variability-driven response observed early during training in Experiment 2 might be driven by variability that stemmed from the presence of EC trials rather than variability stemming from the abruptness of FF onset as modeled in Figure 4. To examine whether this additional variability caused by the EC trials early in Experiment 2 training might account for the overshooting and wrong direction behavior that we observe in Figure 5, we analyzed GF adaptation during training before the first EC trial would have an effect. This allowed us to dissociate the potential effects of EC-induced training period variability from the effects of variability driven by the sudden onset of the training period, because EC-induced training period variability could not be present before the first EC trial was experienced. Specifically, we examined GF adaptation on Trials 2 and 3 of the training period, the first $270^{\circ}$ and $90^{\circ}$ movement direction EC trials, respectively. For the +FF case, we estimated the amount of overshoot by comparing the adaptation levels on these trials to the corresponding asymptotic adaptation for each subject. For the -FF case we estimated the amount of wrong-direction adaptation by comparing the adaptation levels on these trials to the baseline.

\section{Statistical comparisons}

We used paired $t$ tests for all statistical comparisons between adaptation levels and sensitivity estimates across conditions within groups of participants, and unpaired $t$ tests for comparisons between adaptation levels and sensitivity estimates across groups of participants. We used bootstrapping for analyses where we expected the distribution of the data to be highly non-normal. Specifically, we used bootstrapping for ratio estimation, as the ratio between random variables is known to produce a distribution that can be far from normal, even when the random variables themselves are normally distributed (Marsaglia, 1965). We also used bootstrapping for estimating the time constants of task-specific adaptation for GFs and MFs using exponential fits. We chose this method instead of performing a $t$ test between parameters derived from fits to individual subject data, because the high noise in these individual data leads to low confidence about the corresponding individual parameters.

\section{Results}

We began by studying changes in the control of GF and MF when the mean and variability of LF dynamics are systematically manipulated to examine the hypothesis that GF control is more sensitive to the variability than the expected value of LF dynamics. We then examined how variability-driven responses affect the changes in GF and MF control observed after sudden changes in LF dynamics. With this understanding, we investigated whether variability-driven GF adaptation can explain previous results suggesting that GF control adapts faster than MF control (Flanagan et al., 2003). 


\section{Effects of increased environmental variability on GFs and MFs}

While previous work has focused on the adaptive responses of both GFs and MFs to changes in the expected value of environmental dynamics (Flanagan and Wing, 1993, 1997; Shadmehr and MussaIvaldi, 1994; Wolpert and Kawato, 1998; Wolpert et al., 1998; Kawato, 1999; Krakauer et al., 1999; Smith et al., 2006; Danion and Sarlegna, 2007; Sing et al., 2009; Yokoi et al., 2011; Yousif and Diedrichsen, 2012), here we hypothesize that safety margins, which apply to GF but not MF control, are specified based on the variability rather than the expected value of environmental dynamics, leading to fundamental differences between the control of MFs and GFs. In particular, this would predict the control of GFs, but not MFs, to be strongly modulated by environmental variability.

To hold steady against a load, the motor system must apply an MF equal and opposite to the LF: a weaker MF would result in acceleration in the direction of the LF, whereas a stronger MF would accelerate the object against the load. Accordingly, a strategy that yields efficient MF control, minimizing the risk of accelerating toward either direction, would be to exert an MF that continually approximates the expected value of the LF during the course of each movement. This idea is in line with previous work showing that under variable dynamic environments, MFs adapt to the expected value of the perturbation (Scheidt et al., 2001; Takahashi et al., 2001) and that under variable visuomotor transformations, movements adapt to the expected value of the transformation (Burge et al., 2008; Saijo and Gomi, 2012), independent of the amount of variability in both cases.

To apply this strategy to GF control, however, would mean that the planned GF would often be insufficient to maintain grasp because producing a GF equal to the expected value of the required GF (i.e., the expected value of the orthogonal LF divided by the coefficient of friction, $\eta$ ) would result in compromised grasp whenever the required GF exceeds its expected value. This exposes a key dichotomy between the control of GFs and MFs: whereas undercompensating and overcompensating MFs both result in motion errors, undercompensating GFs result in grasp errors while overcompensating GFs do not (Fig. 1). A strategy for improved GF control would thus reduce the risk of slip by programming GFs to be greater than the expected value of the required GF (Johansson and Westling, 1984, 1988; Cole and Johansson, 1993). The specification of the size of this GF safety margin can be viewed as a tradeoff between the increased effort associated a higher margin and the reduced risk of slip that it affords. Here we hypothesize that when GFs are not limited by strength or fatigue, the safety margin for GF control is specified
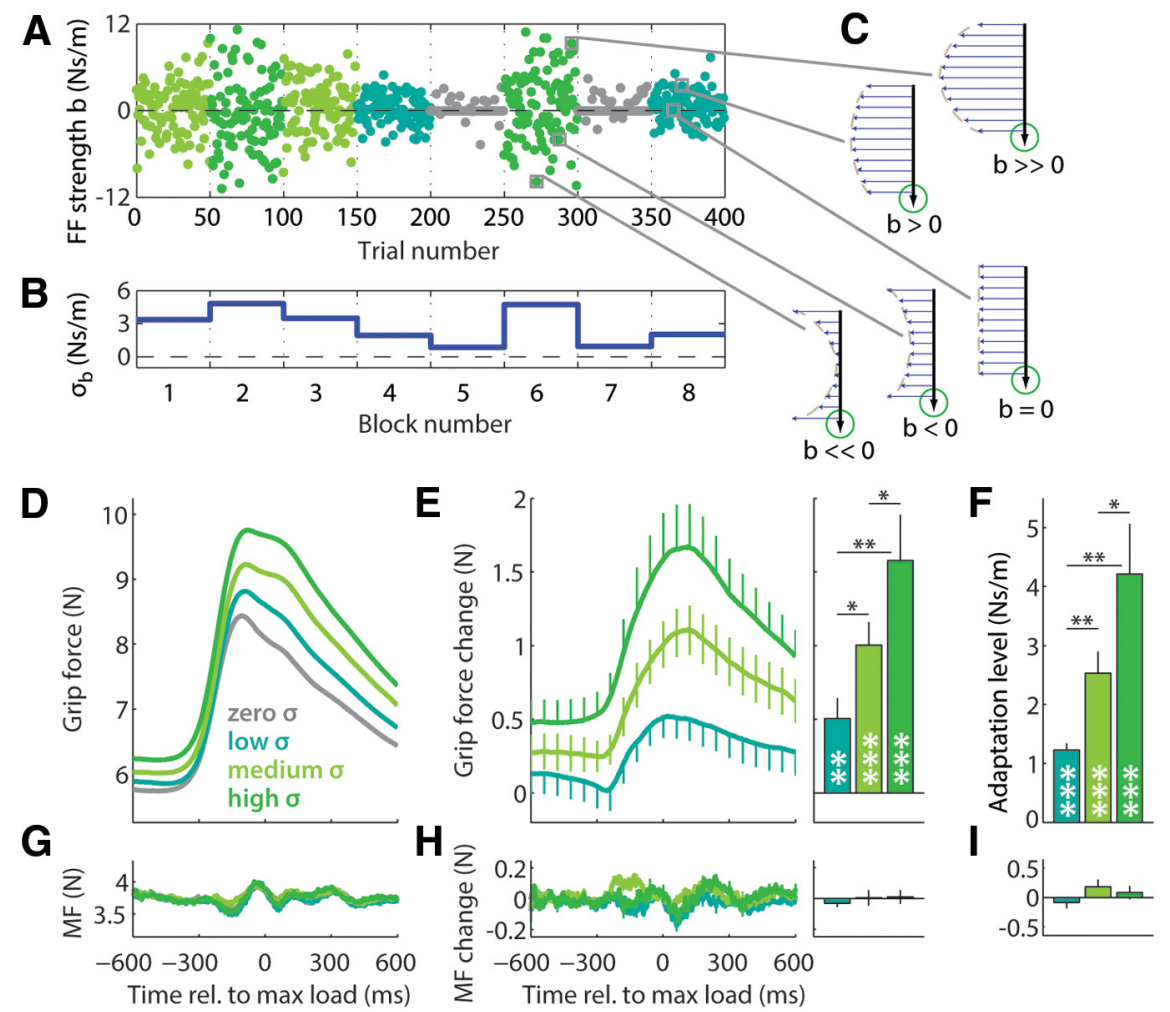

Figure 2. GFs, but not MFs, scale with the level of environmental variability. $\boldsymbol{A}$, Design of Experiment 1a. Following a baseline period (data not shown) participants were exposed to environments that randomly changed in variability from one 50 -trial block to the next, as illustrated. Specifically, the viscosity $(b)$ of a velocity-dependent FF perturbation was drawn from a normal distrilandum applies the forces required to maintain a straight handpath. Using these forces, we estimated the effective $b$ that the robot (see Materials and Methods). $\boldsymbol{B}, S D(\sigma)$ of the values of $b$ for each block, illustrating the four distinct variability the zero- $\sigma$ variability level. Error bars indicate SEM across subjects. The right panel shows the 列 ${ }^{* *} p<0.01,{ }^{* * *} p<0.001$. White asterisks indicate comparisons with the baseline level. Note how the separation between the transient changes in GF during movement, and thus these comparisons do not benefit from the separation between premovement levels suggested in $\boldsymbol{E}$. G-I, Same as $\boldsymbol{D}-\boldsymbol{F}$ but for MF. Note that, similar to $\boldsymbol{E}, \boldsymbol{H}$ shows error bars indicating SEM; however, they are small enough that they are largely obscured by line width and the fluctuations of the MF on the time axis.

based on a fixed level of statistical confidence. Figure 1 illustrates that this hypothesis predicts higher safety margins as uncertainty increases, because the risk of slip at a fixed GF level would increase with higher uncertainty (Fig. $1 E, F$ ).

To investigate the fixed statistical confidence hypothesis, we tested the prediction that the safety margin for GF control would be modulated by environmental variability. We designed a task in which subjects were exposed to environments with different amounts of variability. Subjects performed rapid point-to-point reaching movements while grasping a small object with physical dynamics that changed randomly from one trial to the next, with the amplitude of these random changes systematically varying from one block to the next, as illustrated in Figure $2 A-C$. In this task, the direction of the LF was orthogonal to the grasp axis so that GF levels, rather than finger configuration, determined whether the object slipped (Fig. $1 A, B$ ). In addition to specifying the orientation of grasp relative to the LF, we restricted the possible grasp locations, as freedom to vary the finger configuration 
can influence the applied GF (Fu et al., 2010). Thus, subjects could modulate GF to prevent slip and independently modulate MF to control the motion of the object. After a practice period of 150 trials in which participants were familiarized with the basic task, they were exposed to 1650 -trial blocks with different levels of variability. During each block, the strength $(b)$ of a viscous curl FF environment (Eq. 1) was varied from one trial to the next by drawing $b$ from a Gaussian distribution with mean zero and SD $\sigma$. $\sigma$ was fixed within each block at one of four possible values: $0,1.8$, 3.6, and $5.4 \mathrm{Ns} / \mathrm{m}$, and four blocks were presented for each $\sigma$ value during the 16 -block session, in a randomized order. To give subjects time to adapt to each variability level, we focused our analysis on the last $70 \%$ of the data from each block (see Materials and Methods). It should be noted, however, that the adaptation was fairly rapid and so other choices for the window size yield similar results.

Critically, we applied a leftward static force offset of $4 \mathrm{~N}$ (Eq. 1, $F_{0}$ ) throughout the experiment on top of the time-varying FF perturbation to ensure that the net direction of the force would always remain leftward even for negative values of $b$ as shown in Figure $2 C$. This ensured that negative values of $b$ required smaller GFs than baseline to prevent slip, whereas positive values of $b$ required higher GFs. Without this offset, negative values of $b$ would result in rightward forces, whereas positive values of $b$ would generate leftward forces, with increased GF required to maintain grasp in both cases. Moreover, since load-increasing and load-decreasing values of $b$ were equally likely, any symmetric positive versus negative trial-to-trial learning (Scheidt et al., 2001; Takahashi et al., 2001; Braun et al., 2009) would balance out. As we estimated variability-driven responses based on the average responses during each variability block, the net effect of trial-to-trial learning predicted by current models of motor adaptation (Thoroughman and Shadmehr, 2000; Donchin et al., 2003; Smith et al., 2006; Joiner and Smith, 2008; Lee and Schweighofer, 2009; Sing et al., 2009; Sing and Smith, 2010) should be near zero.

\section{Increased environmental variability leads to increases in GF control but not MF control}

We found that the average GF profiles displayed in each block systematically increased with variability. This is evident in both the raw GF profiles (Fig. 2D) and baseline-subtracted GF profiles, which show the variability-induced changes from the zerovariability baseline blocks (Fig. $2 E$ ). The pattern of variabilityinduced GF change was similar in shape for all nonzero variability levels, but its amplitude grew systematically as $\sigma$ increased. Changes in GF began at movement onset and peaked near the peak velocity point, when the effect of the LF from the viscous curl FF perturbation would be the strongest (due to the velocitydependent FF perturbation). The amplitude of these GF changes scaled with the variability level, peaking at $1.5 \mathrm{~N}$ for the highvariability blocks (Fig. 2E).

To quantify the magnitude of variability-induced changes in both GF and MF, we computed adaptation coefficients for each movement for both GF and MF. These adaptation coefficients were based on linear regressions of the corresponding GF or MF force profile onto the LF profile (Scheidt et al., 2000; Smith et al., 2006; Joiner and Smith, 2008; Gonzalez Castro et al., 2011, 2014; Joiner et al., 2011; Yousif and Diedrichsen, 2012; Sing et al., 2013; Wu et al., 2014; see Materials and Methods). We found that subjects exhibited significantly greater GF adaptation in the low- $\sigma$, medium- $\sigma$, and high- $\sigma$ blocks compared with the zero- $\sigma$ blocks $(p<0.001$ in all three cases, one-tailed paired $t$ tests; Fig. $2 F)$.
Moreover, the GF adaptation was greater in both the medium- $\sigma$ and high- $\sigma$ blocks than the low- $\sigma$ blocks ( $p<0.01$ in both cases), and greater in the high- $\sigma$ blocks than the medium- $\sigma$ blocks $(p<$ 0.05 , one-tailed paired $t$ tests).

In contrast, we found no consistent changes in MF control with variability. The subject-averaged MF profiles were essentially identical for different amounts of variability (Fig. $2 G$ ), and the variability-related changes in MF shown in Figure $2 \mathrm{H}$ were small. Correspondingly, the MF adaptation coefficients were not significantly different from zero (Fig. 2I). In particular, note that the high- $\sigma$ environments display MF levels that are essentially the same as the zero- $\sigma$ environment ( $p>0.48$, two-tailed paired $t$ test; Fig. $2 G, H$ ). These findings indicate that when the mean dynamics are held constant, GF but not MF control systematically depends on the variability of environmental dynamics.

\section{GF control is even more sensitive to changes in the LF variability than the mean $L F$}

Since previous work maintained that GF is controlled based on the expected LF, we wanted to know how the strength of the dependence of GF on LF variability observed in Experiment 1a compares to the dependence of GF on the expected value of the LF. To that end, we estimated the sensitivity (i.e., the slope of the relationship) that GF control displays with respect to both the $\mathrm{SD}$ $\left(\sigma_{\mathrm{LF}}\right)$ and the mean $\left(\mu_{\mathrm{LF}}\right)$ of the LF. Across the four different variability levels (zero, low, medium, and high) in Experiment 1a, we found the relationship between subject-averaged GF levels and $\sigma_{\mathrm{LF}}$ to be approximately linear, with a strong positive correlation between GF levels and $\sigma_{\mathrm{LF}}\left(R^{2}>0.99\right.$; slope $=1.02$; Fig. $3 B$, squares and solid line). When we estimated the $\mathrm{GF} / \sigma_{\mathrm{LF}}$ sensitivity for all 11 individual participants, we found positive slopes for all 11, with an average value of $1.02 \pm 0.21$ (mean \pm SEM across subjects, $p<0.001$, one-tailed paired $t$ test; Fig. $3 B, D$ ). Note that these slopes are all dimensionless because the levels of GF adaptation and $\sigma_{\mathrm{LF}}$ are both quantified in the units of FF strength (newton-seconds per meter).

To put the amplitude of the GF/ $\sigma_{\mathrm{LF}}$ sensitivity into perspective, we estimated the GF/ $\mu_{\mathrm{LF}}$ sensitivity by measuring GF responses to systematic changes in the LF environment. To that end, we presented subjects with a deterministic step change in the environmental dynamics, changing the viscosity coefficient $(b)$ of a viscous curl FF from zero to a constant value of either +7.5 $\mathrm{Ns} / \mathrm{m}$ (Exp. 2a, leftward, $n=18$ ) or $-7.5 \mathrm{Ns} / \mathrm{m}$ (Exp. 2b, rightward, $n=18$ ) for 240 trials following a 160-trial baseline period with $b=0$ (Fig. 3A). Note that, like in Experiment 1a, a leftward static offset force was present throughout to ensure that LFs were reduced in magnitude rather than reversed in direction during rightward $-\mathrm{FF}$ trials (Fig. 2C). We found highly significant and nearly symmetric changes in GF during asymptotic adaptation (last 60 trials) in Experiments $2 \mathrm{a}$ and $2 \mathrm{~b}(2.46 \pm 0.51 \mathrm{Ns} / \mathrm{m}, p=$ 0.0002 and $-2.36 \pm 0.74 \mathrm{Ns} / \mathrm{m}, p=0.0055$, respectively; twotailed paired $t$ tests; Fig. $3 C$ ). These changes in GF correspond to sensitivity estimates (slopes) of $0.33 \pm 0.07$ and $0.31 \pm 0.10$ for individuals in Experiments 2a and 2b, respectively, and $0.32 \pm$ 0.06 for the combined data (Fig. 3C,D). Remarkably, these values are threefold lower than the sensitivity of GF to changes in variability observed in Experiment 1a (1.01 \pm 0.19 ; Fig. 3, compare $B$, $C$ ), indicating LF variability to be a stronger determinant of GF control than mean LF.

We conducted three additional experiments to determine the robustness of the finding that GF control is more sensitive to $\sigma_{\mathrm{LF}}$ than to $\mu_{\mathrm{LF}}$. We first checked whether the stability of GF adaptation in the 50-trial blocks from Experiment 1a was sufficient for 

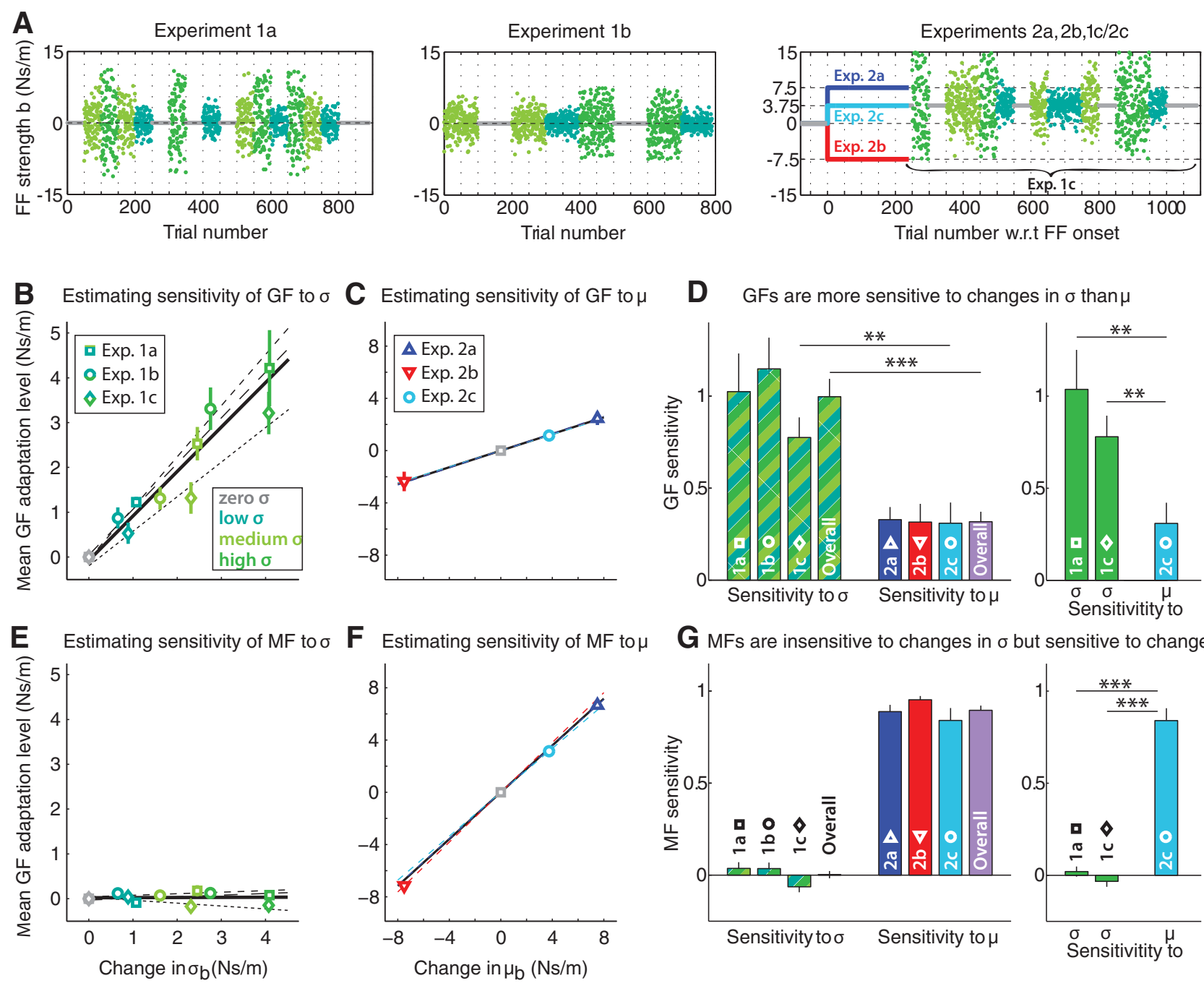

G MFs are insensitive to changes in $\sigma$ but sensitive to changes in $\mu$
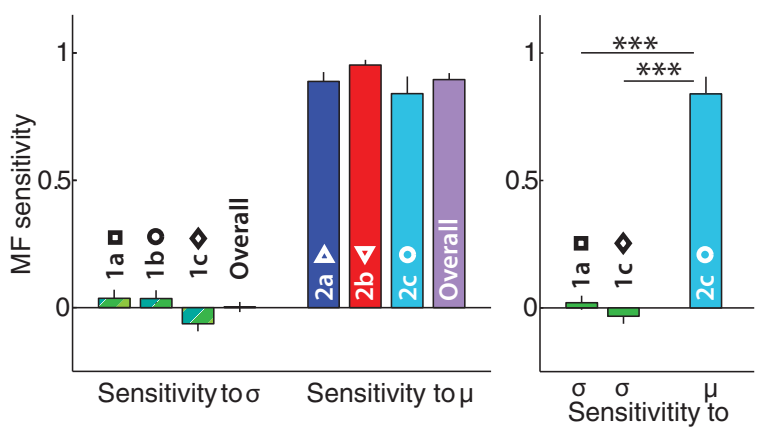

Figure 3. GFs are threefold more sensitive to changes in the variability than changes in the expected value of environmental dynamics. $A$, Comparison between the design of Experiments $1 \mathrm{a}$, $1 \mathrm{~b}$, $2 a, 2 b$, and $1 c / 2 c$. Examples of training schedules are shown. The duration of variability blocks in Experiment $1 b$ is double that of Experiment $1 a$. Experiments $2 a$ and $2 b$ present a step increase or decrease of the FF, respectively. Experiment 1 $1 / 2 c$ begins with a step increase in the FF, which persists for 240 trials (Exp. 2c) and is then followed by the addition of a variable FF component (Exp. 1c) in an analogous fashion to Experiment 1a. Note that, for clarity given the small size of the plots in this panel, the effective FF strength $b$ during EC trials is not shown, in contrast to Figure $2 A$. $\boldsymbol{B}$, Baseline-referenced adaptation levels (mean \pm SEM) for GF for each of the four types of variability blocks and each experiment (1a, 1b, and 1c) plotted against the corresponding increases in the SD of $b$ with respect to the baseline. The black lines indicate linear fits to the data (thin dashed lines, in order of increasing density: Exps. 1a, 1b, and 1c; thick solid line: overall data from all 3 experiments). The three groups display similar slopes for GF versus $\sigma$ and indicate that GF is sensitive to environmental variability. C, Baseline-referenced adaptation levels (mean \pm SEM) for GF for Experiments $2 a, 2 b$, and $2 c$, plotted against the corresponding changes in the mean of $b$ with respect to the baseline. The colored lines indicate linear fits to the data for each experiment (the black line indicates the linear fit to the overall data from all 3 experiments). Note that these fits lie on top of each other, indicating that the sensitivity of GF to the $\mu$ is linear over the range of changes in $\mu$ that we examined. The aspect ratio is the same as in $\boldsymbol{B}$, illustrating that the sensitivity of GF to $\mu$ (i.e., the slope of the fits) is substantially smaller than the sensitivity of GF to $\sigma$ (the slope of the fits in $\boldsymbol{B})$. D, GFs show significantly greater sensitivity to the SD, $\sigma$, compared with the mean, $\mu$, of environmental dynamics. In the left panel, we calculate sensitivities to $\sigma$ based on the slopes in $B$. In the right panel, we calculate sensitivities based on the high-variability blocks from Experiments 1 and $1 c$, where the magnitudes of the baseline-referenced changes in $\sigma$ were similar to magnitude of the baseline-referenced changes in $\mu$ from Experiment $2 c(3.75 \mathrm{Ns} / \mathrm{m}) .{ }^{* *} p<0.01$, ${ }^{* * *} p<0.001$. E-G, Manipulatory forces are not sensitive to changes in the SD, but only to the mean of environmental dynamics. The format of $\boldsymbol{E}-\boldsymbol{G}$ parallels the format of $\boldsymbol{B}-\boldsymbol{D}$.

accurate $\mathrm{GF} / \sigma_{\mathrm{LF}}$ sensitivity estimates. In Experiment $1 \mathrm{~b}$ (Fig. $3 A$ ), variability was modulated from one block to the next like in Experiment 1a, but with longer (100 vs 50 trials) and correspondingly fewer ( 8 vs 16 ) blocks. Experiment $1 \mathrm{~b}$ yielded sensitivity estimates of $1.15 \pm 0.17$ across individuals $\left(p<10^{-6}\right.$, one-tailed paired $t$ test), similar to the ones obtained from Experiment 1a (Fig. $3 B, D$ ), suggesting $\mathrm{GF} / \sigma_{\mathrm{LF}}$ sensitivity estimates to be stable for blocks of 50 trials or greater.

In a second additional experiment (Exp. 2c), we checked whether the GF/ $\mu_{\mathrm{LF}}$ sensitivity estimates from Experiments 2a and $2 \mathrm{~b}$ would be consistent with the sensitivity observed from a smaller change in $\mu_{\mathrm{LF}}$. Because Experiments $2 \mathrm{a}$ and $2 \mathrm{~b}$ were based on changes in $\mu_{\mathrm{LF}}$ with a magnitude $(7.5 \mathrm{Ns} / \mathrm{m})$ that was greater than the $\sigma_{\mathrm{LF}}$ changes examined in Experiments 1a or $1 \mathrm{~b}(\leq 4.1$ $\mathrm{Ns} / \mathrm{m}$ ), examining a smaller change in $\mu_{\mathrm{LF}}$ allowed us to compare $\mathrm{GF} / \sigma_{\mathrm{LF}}$ and $\mathrm{GF} / \mu_{\mathrm{LF}}$ sensitivity estimates using similar displacements for $\sigma_{\mathrm{LF}}$ and $\mu_{\mathrm{LF}}$. This also allowed us to examine the linearity of the relationship between GF and $\mu_{\mathrm{LF}}$. Experiment $2 \mathrm{c}$ mirrored 2a, except that the FF amplitude $(b)$ was halved $(+3.75$ vs $+7.5 \mathrm{Ns} / \mathrm{m}$ ). The results showed an asymptotic adaptation level in the last 60 trials of the 240-trial FF exposure that was almost exactly half of that observed in Experiment $2 \mathrm{a}$ (1.16 \pm 0.42 vs $2.46 \pm 0.51 \mathrm{Ns} / \mathrm{m}$ ). This corresponds to nearly identical GF/ $\mu_{\mathrm{LF}}$ sensitivity estimates for Experiments $2 \mathrm{a}, 2 \mathrm{~b}$, and $2 \mathrm{c}$ 
$(0.33 \pm 0.07,0.31 \pm 0.10$, and $0.31 \pm 0.11 \mathrm{Ns} / \mathrm{m}$, respectively $)$, suggesting a fixed level of $\mathrm{GF} / \mu_{\mathrm{LF}}$ sensitivity up to at least 7.5 $\mathrm{Ns} / \mathrm{m}$, in line with a linear relationship between GF and $\mu_{\mathrm{LF}}$. Comparison of the GF/ $\sigma_{\mathrm{LF}}$ sensitivity estimates from the high- $\sigma$ blocks in Experiment 1a and the GF/ $\mu_{\mathrm{LF}}$ sensitivity estimate from $2 \mathrm{c}$ revealed the former to be significantly greater than the latter $(1.04 \pm 0.21$ vs $0.31 \pm 0.11, p=0.0043$, onetailed unpaired $t$ test; Fig. $3 D$, right). This is in line with the idea that GF is substantially more sensitive to the variability than the expected value of the LF environment, even when displacements for $\sigma_{\mathrm{LF}}$ and $\mu_{\mathrm{LF}}$ are similar $(4.1$ and $3.75 \mathrm{Ns} / \mathrm{m}$, respectively).

In a third additional experiment (Exp. 1c), we checked whether the GF/ $\sigma_{\mathrm{LF}}$ sensitivity estimates from Experiments 1a and $1 \mathrm{~b}$ would be consistent with the sensitivity observed when GF variability was centered around a nonzero mean. We performed this experiment immediately after Experiment $2 \mathrm{c}$ in the same session with the same participants, which also enabled us to compare $\mathrm{GF} / \sigma_{\mathrm{LF}}$ and $\mathrm{GF} / \mu_{\mathrm{LF}}$ sensitivity estimates in the same participants. Note that we used a value of $+3.75 \mathrm{Ns} / \mathrm{m}$ for $\mu_{\mathrm{LF}}$ in $1 \mathrm{c}$ to maintain continuity with the FF training in 2c. Again, we found GF to be more sensitive to $\sigma_{\mathrm{LF}}$ than $\mu_{\mathrm{LF}}(0.78 \pm 0.11$ vs $0.31 \pm$ $0.11, p=0.0013$, one-tailed paired $t$ test; Fig. $3 D$, right), consistent with our previous findings. Also consistent with our previous findings, we found the GF/ $\sigma_{\mathrm{LF}}$ sensitivity in Experiment $1 \mathrm{c}$ to be similar to that from Experiments $1 \mathrm{a}$ and $1 \mathrm{~b}$ in that an ANOVA revealed no significant differences in $\mathrm{GF} / \sigma_{\mathrm{LF}}$ sensitivity across experiments $\left(F_{(2,48)}=1.46, p=0.24\right)$.

Analogous analysis of the MF data from Experiments $2 a, 2 b$, and $2 \mathrm{c}$ revealed highly significant asymptotic adaptation of MF levels to changes in $\mu_{\mathrm{LF}}$ [Exp. $2 \mathrm{a}(b=+7.5 \mathrm{Ns} / \mathrm{m}): 6.66 \pm 0.27$ Ns/m; Exp. 2b $(b=-7.5 \mathrm{Ns} / \mathrm{m}):-7.14 \pm 0.15 \mathrm{Ns} / \mathrm{m}$; Exp. 2c $(b=+3.75 \mathrm{Ns} / \mathrm{m}): 3.15 \pm 0.25 \mathrm{Ns} / \mathrm{m}, p<10^{-6}$ in all three cases, two-tailed paired $t$ tests; Fig. $3 F]$. Note the nearly symmetric increases and decreases in the adaptive responses for Experiments $2 \mathrm{a}$ and $2 \mathrm{~b}$. These MF levels correspond to $\mathrm{MF} / \mu_{\mathrm{LF}}$ sensitivity estimates of $0.89 \pm 0.04,0.95 \pm 0.02$, and $0.84 \pm 0.07$, for Experiments $2 \mathrm{a}, 2 \mathrm{~b}$, and $2 \mathrm{c}$, respectively (Fig. $3 G$ ). The similarity between these estimates corresponds to the nearly linear relationship between MF and $\mu_{\mathrm{LF}}$ observed in Figure 3F. In contrast, the overall $\mathrm{MF} / \sigma_{\mathrm{LF}}$ sensitivity we observed in Experiments $1 \mathrm{a}, 1 \mathrm{~b}$, and $1 \mathrm{c}$ was much smaller and not different from zero $(0.002 \pm 0.020, p>0.9$, two-tailed paired t test; Fig. $3 E, G)$ in line with the lack of MF modulation observed in Figure $2 G-I$ when variability was increased.

\section{GF control maintains a safety margin based on statistical confidence}

Remarkably, our findings show not only that GF control is sensitive to the SD of environmental dynamics, but that this sensitivity is higher $\left(p<10^{-6}\right.$, two-tailed unpaired $t$ test between Exps. 1a, 1b, and 1c vs Exps. 2a, 2b, and 2c) than the sensitivity to the environment's mean dynamics as illustrated in Figure 3 ( $2.82 \pm 0.27$-fold higher, estimated using bootstrap when combining the data from Exps. 1a, 1b, 1c, 2a, 2b, and 2c). Since the $\mathrm{GF} / \sigma_{\mathrm{LF}}$ sensitivity acts to scale the GF safety margin against slip by the LF variability, the ratio between the $\mathrm{GF} / \mu_{\mathrm{LF}}$ sensitivity and the $\mathrm{GF} / \sigma_{\mathrm{LF}}$ sensitivity can be used to determine the statistical confidence associated with the observed safety margin (see Materials and Methods). In particular, the ratio of $2.82 \pm 0.27$ that we observed corresponds to GF control in a variable environment that is $2.82 \mathrm{SDs}$ above that required for the expected value of dynamics. For normally distributed environmental perturba- tions, like the ones administered in Experiments 1a, 1b, and 1c, this corresponds to a $z$-score of $2.82 \pm 0.27$, which amounts to $>99 \%$ confidence interval against slip. Unfortunately, the precise frequency of slippage incidents is difficult to measure experimentally as these incidents usually result in microslips, which subjects rarely notice and often correct almost instantaneously (Johansson and Westling, 1984, 1987). In fact, we were not able to detect any instances of loss of grasp from slip during movement in our experiments. The low likelihood of overt slippage we observed is consistent with the high statistical confidence against slip that we report here.

\section{A computational model for understanding how GF control responds to both the mean and variability of LF dynamics}

We next investigated how the adaptation of GF levels to environmental variability might manifest following a sudden but enduring change in the environment as in Experiment 2. We hypothesized that the motor system might estimate the current levels of the mean and the variability of the LF environment based on a window of recent experience. If this is the case, a recent sudden change in the environment should increase the motor system's estimate of variability because some trials in the window would be at the previous perturbation level and others would be at the new perturbation level. This increase should wane after prolonged exposure when most or all of the trials in the recent memory window would be associated with the new environment. The ACEVU model, illustrated in Figure 4, posits that the overall adaptive response simply reflects the sum of a mean-driven and a variability-driven component, based on windowed estimates of the expected value and the variance of the environment.

While the exact shape of the memory window is not crucial for the model, we used an exponentially decaying memory window to estimate both the mean and the variability of the environment because it describes an estimation process that weighs past experiences less and less as they become more distant. This window shape is also compatible with linear state-space models of trialby-trial adaptation with finite retention (retention coefficients $<1$ ), and it captures the approximately exponential learning curves that have been observed for motor adaptation. Moreover, a recent study found evidence that subjects track the variance of a visuomotor perturbation using an exponential memory window (Landy et al., 2012).

On-line estimates of the mean and variability of the environment for the ACEVU model in response to a step change in the environment are illustrated in Figure 4. Following the step, the mean estimate changes monotonically, asymptotically approaching a level appropriate for the new environment (Fig. 4A) in a symmetric manner that is specific for positive and negative steps. In contrast, the variability estimate increases transiently as discussed above, but in an identically positive manner after either step change (Fig. 4B). Thus a critical feature of the ACEVU model is that the mean-driven component of GF adaptation produces opposite responses for opposite changes in the environment (i.e., positive vs negative $\mathrm{FFs}$ ), whereas the variability-driven component produces identical responses, which cannot be specific to the direction of the step change. This is the case because the variance of a signal is equal to the variance of its opposite. Figure $4 C, D$ shows how estimates of the expected value and variability would combine in contributing to the adaptation of GF and MF throughout the time course of this adaptation. In these predictions, MFs and GFs both respond to changes in the mean of the environment as previous studies have shown (Flanagan and Wing, 1993, 1997; Scheidt et al., 2001; Takahashi et al., 2001; 
A Running estimate of $\mu \quad$ B

Running estimate of $\sigma$
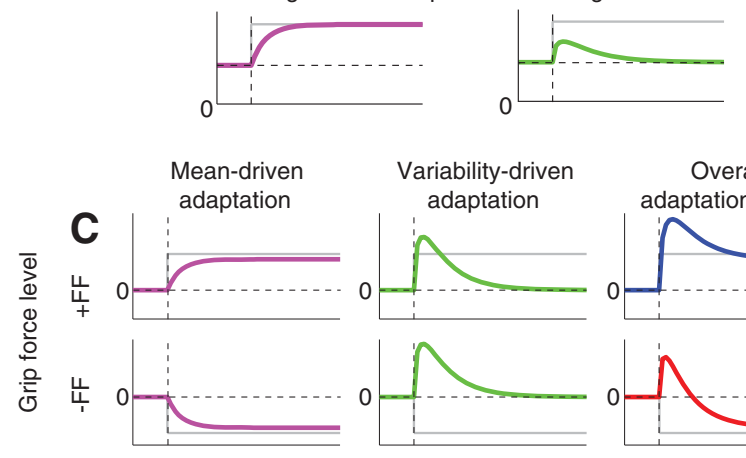

Overall adaptation (sum)

\section{$\overline{0}$
$\frac{1}{0}$
0
0
0
$\frac{1}{2}$
$\frac{1}{0}$
$\frac{0}{5}$
$\frac{0}{2}$
$\frac{0}{2}$
$\frac{\pi}{2}$}
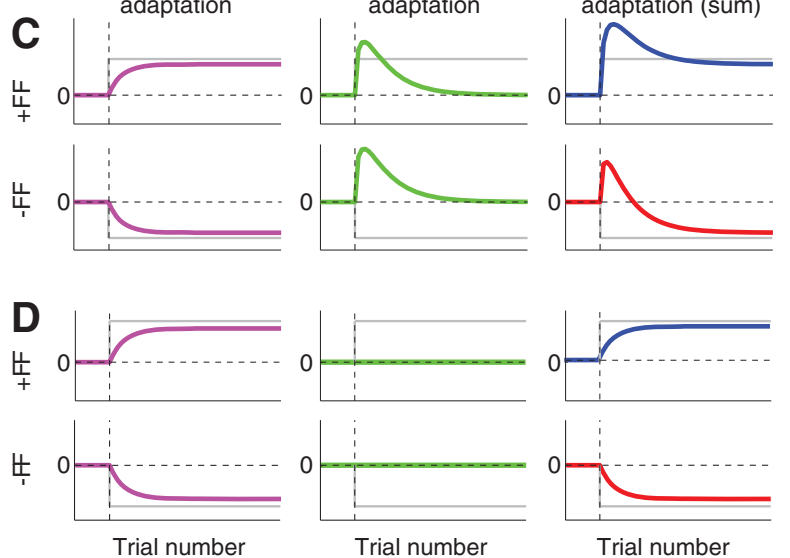

- Perturbation $=$ Predicted adaptation for $+F F=$ Predicted adaptation for $-\mathrm{FF}$

Figure 4. Predictions of a model for the adaptation of GFs and MFs based on distinct meandriven and variability-driven components. $\boldsymbol{A}, \boldsymbol{B}$, Running estimates of mean and variability during a step change in environmental dynamics. These estimates weigh recent memories more strongly than older ones using an exponentially shaped memory window. The mean estimate $(\boldsymbol{A})$ will tend monotonically toward the post-step value, whereas the SD estimate $(\boldsymbol{B})$ will transiently increase, rising rapidly following the step change and then receding as memories that include the prestep environment fade away. $\boldsymbol{C}$, Mean-driven and variability-driven components of the adaptive GF response following a positive (+FF) or negative ( $-F F)$ change in environmental dynamics. Critically, whereas the mean-driven component will follow positive or negative changes in environmental dynamics, the variability-driven component will be positive for changes in either direction. Thus our ACEVU model, which incorporates these two components, predicts asymmetrical, nonmonotonic GF adaptation curves. A positive FF would elicit overshooting adaptation (blue) due to complementary mean-driven and variabilitydriven components, whereas a negative FF would elicit bimodal adaptation (red) due to opposing mean-drive and variability-driven components. D, Same as $\boldsymbol{C}$ but for MFs. As MFs are insensitive to the variability of environmental dynamics, the variability-driven component will be zero throughout adaptation, leading to monotonic adaptation curves resulting in predictions of symmetrical, monotonic adaptation for the ACEVU model for both positive and negative changes in environmental dynamics.

Mawase and Karniel, 2012); however, only GFs respond to changes in the variability, based on the results of Experiment 1.

\section{Uncertainty versus variability}

Our instantiation of the ACEVU model (Eq. 2) uses a running estimate of variability to estimate the amount of uncertainty about environmental dynamics. Variability and uncertainty are closely related: for example, the systematic block-to-block manipulation of variability in Experiments $1 \mathrm{a}-1 \mathrm{c}$ results in corresponding changes in the uncertainty about the LFs likely to be encountered in the environment. It should be noted, however, that variability and uncertainty, though related, are distinct qualities. Variability is easy to rigorously and specifically define as the statistical variance around the mean of the data. However uncertainty is trickier: it is related to the unpredictable part of the variability. Thus, to estimate uncertainty, the properties of the predictor must be understood. This would allow uncertainty to be defined as the statistical variance of the difference between actual and predicted values of the environment rather than the variance of actual values alone. The running estimate of the mean experienced load in the ACEVU model can be seen as a LF pre- diction relative to which environmental variability is computed. This bases the ACEVU model on an estimate of uncertainty rather than the variability around a fixed mean. In fact, neither the width of the LF prediction window nor the form of the prediction itself (i.e., using a predictor not based on an exponential time window) need be analogous to that for variability estimation as in the instantiation of the model in Equation 2, highlighting the dichotomy between LF prediction and the estimation of uncertainty around that prediction.

\section{The ACEVU model predicts nonmonotonic adaptation}

The superposition of a specific $\mu$-driven and a nonspecific $\sigma$-driven component of the adaptive response in the ACEVU model results in a series of counterintuitive predictions for GF adaptation. In combining separate contributions from $\mu$-driven and $\sigma$-driven adaptation, our model predicts that positive perturbations (increases in LF) will yield adaptation curves that rise especially rapidly but overshoot their asymptotic levels before settling (Fig. 4C). According to the model, the rapid rise will occur because during initial adaptation, the task-specific $\mu$-driven and task-nonspecific $\sigma$-driven components will both contribute to an increase in GF levels. However, the $\sigma$-driven contribution will fall off as training proceeds, and an overshoot will result if this fall off outpaces the continued rise of the task-specific $\mu$-driven component.

Perhaps even more counterintuitive is a second prediction also illustrated in Figure 4C: that decreases in LF will initially lead to increases in GF. This will occur if the increase in GF due to the nonspecific $\sigma$-driven component of adaptation to a negative perturbation outweighs the reduction in GF due to the task-specific $\mu$-driven component. Prolonged training with a decreased LF will then eventually lead to a reduction of GF levels toward those appropriate for the environment as the nonspecific $\sigma$-driven component subsides. Thus the ACEVU model remarkably predicts an initial adaptive response in the "wrong" direction followed by a reversal resulting in appropriately decreased GF levels when decreased LFs are encountered.

Unlike GF control, nonspecific $\sigma$-driven adaptation should not contribute to MF control according to the results of Experiment 1, which show MF to be insensitive to $\sigma_{\mathrm{LF}}$. Consequently our model predicts that MF adaptation curves will be fully determined by taskspecific $\mu$-driven adaptation and thus be monotonically directed toward an asymptotic level appropriate for each new environment be it positive or negative as shown in Figure 4D.

\section{GFs adapt to an additive combination of uncertainty and the expected value}

To examine how GFs and MFs actually evolve following a sudden change in physical dynamics, we analyzed the full time course of the adaptation data from Experiment 2. For the leftward +FF group, we found that the GF learning curve displayed a clear overshoot. It reached a maximum value at $\sim 10$ trials after exposure before settling at a significantly lower asymptotic adaptation level as shown in the blue GF profiles in Figure $5 A, B$ and the blue learning curve in Figure 5C (adaptation levels of $7.90 \pm 1.15$ $\mathrm{Ns} / \mathrm{m}$ vs $2.46 \pm 0.51 \mathrm{Ns} / \mathrm{m}$ for the first 20 trials of adaptation vs the last $60, p<0.001$, two-tailed paired $t$ test; Fig. $5 D$ ). Individually, 16 of the 18 participants in the $+\mathrm{FF}$ group displayed higher average GFs in the first 20 trials than in the last 60 of the FF training epoch.

For the rightward -FF group, the initial adaptation resulted in seemingly inappropriate increases in GF above baseline, which persisted for $\sim 40$ trials before the adaptive responses appropri- 

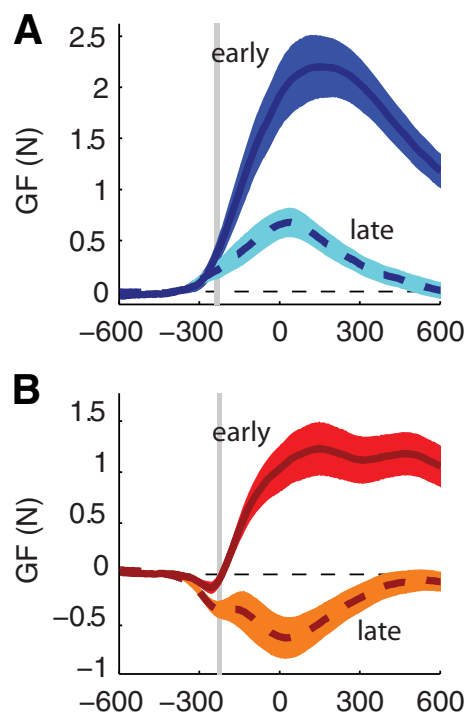

Time relative to peak velocity (msec)
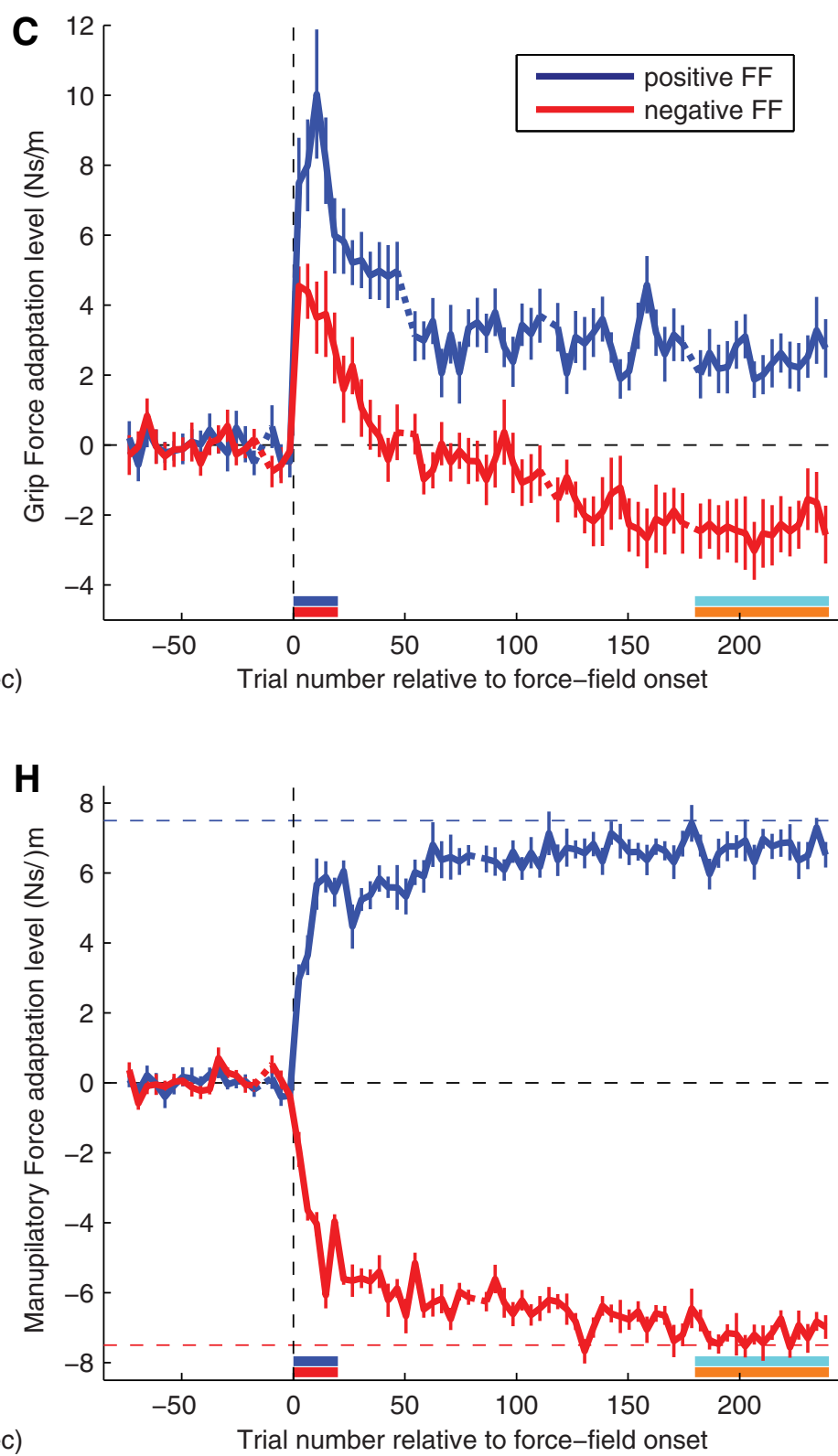
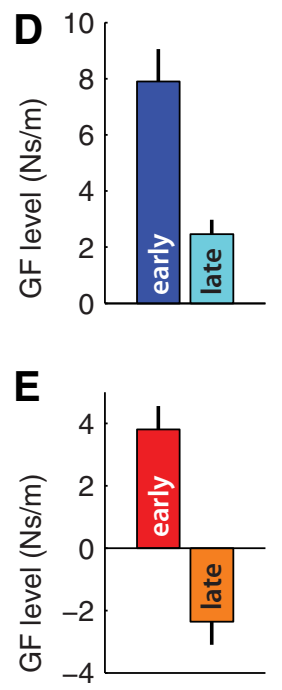
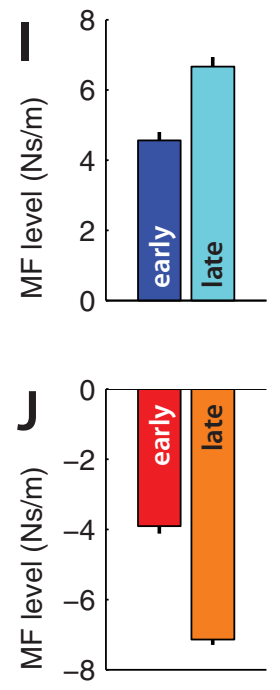
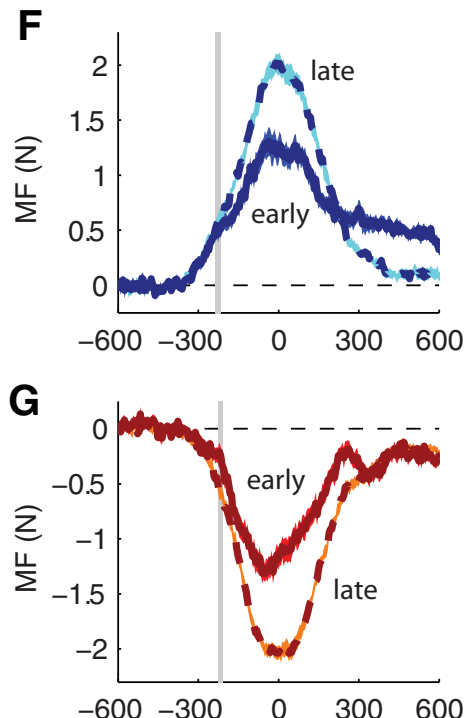

Time relative to peak velocity $(\mathrm{msec})$

Figure 5. GFs, but not MFs, exhibit asymmetric, nonmonotonic adaptation curves following opposite perturbations. $A, B$, Baseline-referenced GF profiles early and late (mean of first 20 vs last 60 trials) following the onset of positive ( $\boldsymbol{A}$, leftward) and negative ( $\boldsymbol{B}$, rightward) FF perturbations. Note that in $\boldsymbol{B}$, the early response is opposite of what is appropriate for the applied $\mathrm{FF}$, whereas the late response is appropriately directed. The shaded areas represent SEM across subjects. The gray vertical line represents the $95 \%$ confidence interval for movement onset. $C$, Corresponding GF adaptation curves based on the adaptation coefficient (see Materials and Methods). Each datapoint in the adaptation curve represents a bin of four trials in each of the two movement directions (8 movements in total). GFs initially increase in both FFs, but eventually reach asymptote at levels that are approximately equal and opposite $(2.46 \pm 0.51 \mathrm{Ns} / \mathrm{m}$ for the $+\mathrm{FF}$ and $-2.36 \pm 0.74 \mathrm{Ns} / \mathrm{m}$ for the $-\mathrm{FF}$ ). This corresponds to overshooting adaptation for the $+\mathrm{FF}$ group and bimodal adaptation for the $-\mathrm{FF}$ group as predicted by the model in Fig $4 \mathrm{C}$. The colored horizontal lines in the bottom of the graph indicate the learning periods shown in $A$ and $B$ : early training (first 20 trials, blue for $+F F$ and red for $-F F$ ) and late training (last 60 trials, cyan for $+F F$ and orange for $-F F$ ). $\boldsymbol{D}, \boldsymbol{E}$, GF levels for the early and late (mean of first 20 vs last 60 trials) stages of adaptation. $\boldsymbol{F}-\boldsymbol{J}$, Same as $\boldsymbol{A}-\boldsymbol{E}$ but for MF, illustrating symmetric, monotonic adaptation patterns as predicted by the model in Fig. 4D.

ately decreased below baseline levels, where they slowly settled to asymptote as shown in Figure 5C. Correspondingly, the adaptation level during the first 20 trials was significantly greater than baseline, whereas the adaptation level during the last 60 was significantly lower than baseline as shown in the red GF profiles in Figure $5 A, B$ and the red learning curve in Figure $5 C$ (adaptation levels of $+3.80 \pm 0.75 \mathrm{Ns} / \mathrm{m}$ vs $-2.36 \pm 0.74 \mathrm{Ns} / \mathrm{m}, p<0.0001$ and $p<0.01$, respectively, two-tailed paired $t$ tests; Fig. $5 E$ ). Individually, the GF level was higher than baseline in 16 of 18 subjects during the first 20 trials, and lower than baseline in 15 of 18 subjects during the last 60 trials. Both the direction reversal observed in the -FF data (red) and the pattern of overshoot and subsequent fall off observed in the +FF data (blue) were remarkably well predicted by the ACEVU model displayed in Figure 4, which combines nonspecific $\sigma$-driven adaptation and FF-specific $\mu$-driven adaptation.

It should be noted that EC trials were interspersed with FF trials during training, perhaps complicating the findings. These trials are less disruptive to training than catch trials on which FF perturbations are withheld (Thoroughman and Shadmehr, 2000; Smith and Shadmehr, 2005) because motor errors that drive learning are held near zero for EC trials in contrast to the large 
errors often associated with catch trials. However to hold lateral errors near zero, an external force must be applied by the robot arm on EC trials, which effectively cancels the lateral force output of the learner. Because learning is generally partial, this external force pattern can be viewed as a certain fraction of the force level applied during FF trials, introducing some variability in the effectively applied FF between FF and EC trials, and thus perhaps increasing variability-driven adaptation in line with the ACEVU model. Thus, the increased variability-driven response early during training in Experiment 2 might be driven by variability that stemmed from the presence of EC trials rather than variability stemming from the abruptness of FF onset as modeled in Figure 4 . However, when we examined our data to determine whether this was the case, we found evidence for both key predictions of the ACEVU model even before the first EC trial could affect the variability estimate: a GF overshoot in the +FF group $(5.6 \pm 2.3 \mathrm{Ns} / \mathrm{m}$ overshoot, $p=$ 0.012 ) and adaptation toward the wrong direction in the $-\mathrm{FF}$ group $(+5.1 \pm 1.3$ $\mathrm{Ns} / \mathrm{m}, p=0.0006)$. This indicates that the overshooting and wrong direction learning we observe do not require the presence of EC trials. However, we cannot rule out the possibility that the amplitude of these effects is exaggerated by it.

\section{MFs adapt chiefly to the expected value of environmental dynamics}

In contrast to GF adaptation, we found MF adaptation to be characterized by highly symmetric, monotonic responses to increases and decreases in LF, as evidenced by the MF learning curves displayed in Figure $5 \mathrm{H}$ and the force profiles displayed in Figure $5 F, G$. Accordingly, the amplitude of the average MF adaptation level during the first 20 trials was significantly lower in magnitude than the average adaptation level during trials $181-240$ (leftward FF: $4.56 \pm 0.24 \mathrm{Ns} / \mathrm{m}$ vs $6.66 \pm$ $0.27 \mathrm{Ns} / \mathrm{m}, p<10^{-4}$; rightward FF: $-3.92 \pm 0.21 \mathrm{Ns} / \mathrm{m}$ vs $-7.14 \pm 0.15 \mathrm{Ns} / \mathrm{m}, p<10^{-6}$, two-tailed paired t-tests; Fig. $5 I, J)$. The highly symmetric learning curves indicate that there is little nonspecific adaptation, with MF adaptation highly specific to the experienced environment. This is consistent with the idea that the nonspecific component of the adaptive response arises from $\sigma$-driven adaptation, which we found to be minimal for MF in Experiment 1.

\section{Robustness of results using different metrics}

To ensure that our observations were not specifically related to the adaptation coefficient metric we used to quantify FF adaptation, we repeated the analysis using an even simpler metric: the GF observed when the effect of $b$ on the LF produced by the imposed environment was maximal (Sing and Smith, 2010; Pekny et al., 2011; Joiner et al., 2013). We found that the learning curves derived from this measure were essentially identical in shape to those displayed in Figure $5 C, H\left(R^{2}>0.97\right.$ for the $+\mathrm{FF}$ and $-\mathrm{FF}$ GF learning curves and for the $+\mathrm{FF}$ and $-\mathrm{FF}$ MF learning curves).

\section{Decomposition of GFs into perturbation-specific and perturbation-nonspecific components}

Based on the ACEVU model, task-specific adaptation should differentially affect the adaptive responses to two opposite perturbations, whereas the task-nonspecific adaptation will be common to both. We used this fact to decompose the experimentally observed adaptive responses to the $+\mathrm{FF}$ and $-\mathrm{FF}$ environments into specific (differential) and nonspecific (common) components (see Materials and Methods). Specifically, we applied this differential versus common component analysis to the GF profiles $[\mathrm{GF}(t)]$ in Experiment 2 to determine the specific and nonspecific components of these profiles (Fig. 6A,C). This analysis reveals that over the course of adaptation, the amplitude of the nonspecific common-mode GF profile rises from baseline during initial adaption and wanes as adaptation proceeds. Despite the 
A specific and non-speficic adaptation curves for GF $\mathbf{C}$

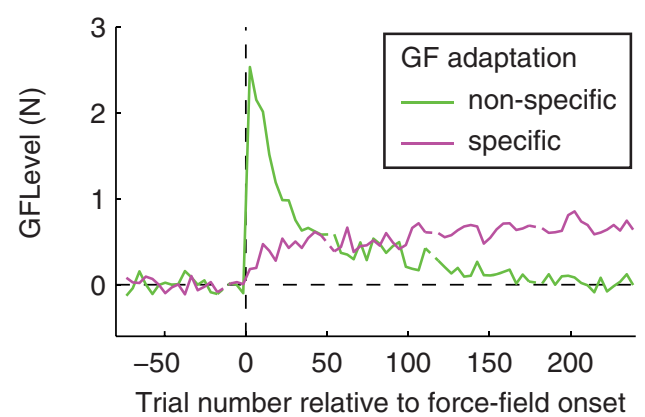

B Specific and non-speficic adaptation curves for MF

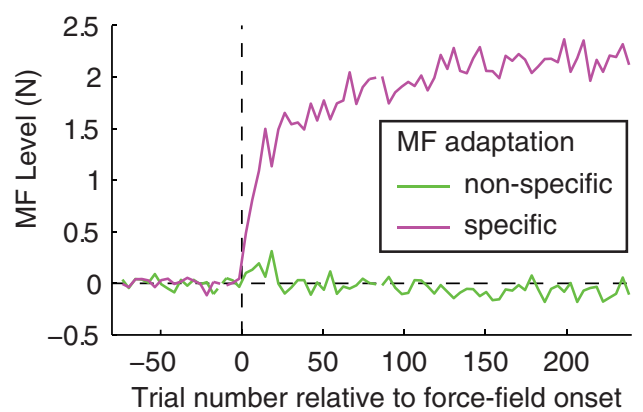

Specific adaptation evolves with the same timescale for both GF and MF
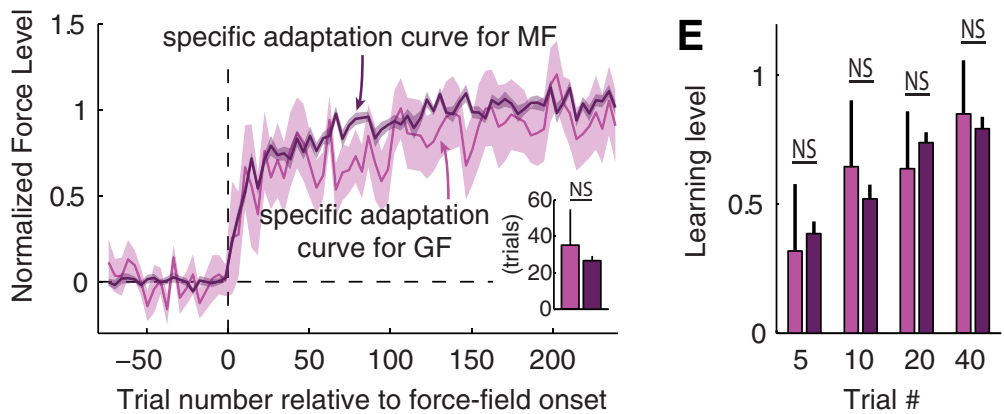

Non-specific adaptation differs for GF and MF

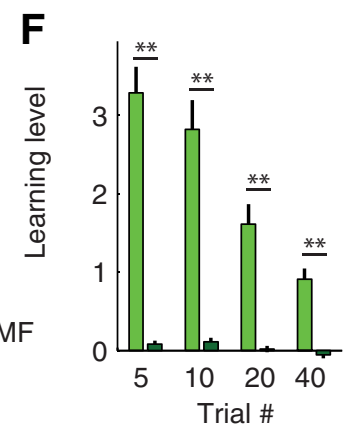

Figure 7. Adaptation curves for specific and nonspecific components. $\boldsymbol{A}, \boldsymbol{B}$, Estimates of the specific (magenta) and nonspecific (green) components of the learning curves for GF ( $\boldsymbol{A})$ and $\mathrm{MF}$ ( $\boldsymbol{B}$ ). The shapes of these components match the predictions in Figure 4C,D.C, Comparison of the learning curves for the specific components of GF and MF, showing that they display similar time courses of adaptation. Adaptation curves were normalized by the overall asymptotic adaptation level (last 60 trials). Shading indicates SEM across subjects. The inset shows the time constants of adaptation for these two specific components (mean \pm SEM estimated using bootstrap). D, Same as ( but for the nonspecific component. E, Comparison of the adaptation levels for the specific components of GF (light) and MF (darker) in five-trial windows centered around trials $5,10,20$, and 40 . None of the differences are significant ( $p>0.5$ in all cases). $F$, Same as $\boldsymbol{E}$ but for the nonspecific component. All differences are highly significant $\left(p<10^{-6}\right.$ in all cases).

changes in amplitude, the shape of the common-mode GF profile remains remarkably constant as training progresses (Fig. 6A, $B$, colored lines). The extent of this shape consistency is apparent when amplitude-normalized force profiles are compared with each other in Figure 6B. Critically, this shape is strikingly similar to the shape of the GF profiles observed in Experiment 1a, when the effects of environmental variability were measured $\left(R^{2}\right.$ values between 0.92 and 0.99 for the six common-mode profiles shown normalized in Fig. $6 B$, and $R^{2}=0.98$ for the mean of these). Note that the GF profile from Experiment la is shown as the dark gray dashed line in Figure $6 \mathrm{~B}$ for reference. The close match between the shapes of the force profiles for the transiently occurring common-mode component of the step response from Experiment 2 and the variability-driven adaptive response directly measured in Experiment la is consistent with the idea that the transient nonspecific component the GF learning curve reflects the motor system's estimate of environmental variability as predicted by the ACEVU model.

On the other hand, the task-specific differential mode component of the GF profile assumes a shape very close to the shape of the viscous LF profile (Fig. $6 C, D$ ). This similarity increases with training, with $R^{2}$ values increasing to 0.91 by the end of the training period $\left(R^{2}=0.93\right.$ for the average profile). Thus the shapes of the force profiles associated with the specific and nonspecific components of the GF adaptation data from Experiment 2 were more closely matched to the mean LF profiles observed in Experiments $2 \mathrm{a}$ and $2 \mathrm{~b}$ and the variability-driven changes in GF observed in Experiment 1a, respectively, than to each other $\left(R^{2}\right.$ values of 0.93 and 0.98 , respectively, vs an $R^{2}$ of 0.50 ). This finding provides direct experimental support for the theoretical links posited in the ACEVU model between task-specific adaptation and expected value estimation on one hand and between tasknonspecific adaptation and variability estimation on the other.

\section{Learning curves for the specific and nonspecific components of GF and MF adaptation}

We then leveraged our knowledge about the shapes of the force profiles associated with the nonspecific (Fig. $6 A, B$ ) and taskspecific (Fig. $6 C, D$ ) components of adaptation to estimate the learning curves associated with each component. Instead of the standard practice of singly regressing the adaptive responses onto the shape of the viscous load force profile to estimate an adaptation coefficient, as in Figure 5, we simultaneously regressed the adaptive responses we observed onto the shapes of both the common-mode and differential-mode components (see Materials and Methods), to obtain learning curves for nonspecific (green) and task-specific (purple) adaptation, respectively, for both GF (Fig. 7A) and MF (Fig. 7B). This analysis reveals that the task-specific differential-mode component of adaptation (purple) displays a monotonic increase toward an asymptote, following a similar time course for both GF and MF (Fig. $7 C$ for a direct comparison). In contrast, the task-nonspecific common-mode component (green) for the GF is transient (it displays a rapid increase toward a sharp peak and then a gradual decay toward baseline) and present only during GF adaptation. This is consistent with the mirror-opposite MF learning curves observed for 
the leftward and rightward FF groups in Figure 5I, which suggest fully-specific MF learning, and with the finding in Experiments 1a and $1 \mathrm{~b}$ that MFs are unrelated to environmental variability. Remarkably, we find that the shapes of these task-specific and task-nonspecific components of the GF learning curves (Fig. $7 A, B$ ) closely match the predictions of the ACEVU model (Fig. 4) for distinct mean-driven and variability-driven responses based on exponentially windowed running estimates of these quantities.

The decomposition of the learning curves into task-specific and task-nonspecific components provides the opportunity to compare the rate of task-specific GF and MF adaptation. We observed a strong similarity between the learning curves for the task-specific (purple) adaptation for GF and MF ( $r=0.84)$, suggesting similar adaptation rates (Fig. $7 C$, which compares asymptote-normalized task-specific learning curves). Correspondingly, fitting single exponentials to these learning curves revealed no difference between the time constants for taskspecific GF versus MF adaptation ( $35.1 \pm 19.6$ vs $26.6 \pm 2.6$ trials, $p=0.334$, estimated using bootstrap; Fig. 7C, inset). Moreover, we found no differences between normalized adaptation levels for GF and MF at trials 5, 10, 20, and 40, estimated using five-trial bins $(~ p>0.5$ in all cases, two-tailed paired $t$ test; Fig. $7 E)$. Together these findings indicate that the FF-specific components of GF and MF adapt at essentially the same rate.

\section{Discussion}

The current results show that the variability of LF dynamics plays a crucial role in GF control, in contrast to previous work, which primarily focused on how the predictive control of GF depends on the expected value of environmental dynamics. In Experiment 1 , we found that modulating LF variability has no effect on MF control but can drive large, systematic changes in GF control (Fig 2). In fact, we found GF control to be threefold more sensitive to the SD of the environment than its mean (Fig 3 ), in line with providing 3- $\sigma$ statistical confidence against slip and suggesting that variability-driven effects may play an even more important role in GF control than the expected value of the load.

We next examined how variability-driven GF control might affect the responses to sudden deterministic (step) changes in the environment. The ACEVU model, which instantiates our hypothesis that GF adaptation is based on a superposition of distinct adaptive responses to the mean and variability of the LF environment, predicts surprisingly unusual asymmetric and nonmonotonic learning curves following sudden changes in load (Fig. 4). Experimental data directly confirm these unusual predictions, demonstrating adaptation that begins in the wrong direction when the load decreases, and overshoots its asymptote when the load increases (Fig. 5). We used ACEVU to specifically decompose GF adaptation into the force profile components associated with $\sigma$-driven and $\mu$-driven learning and to determine the corresponding learning curves (Figs. 6, 7). Interestingly, the distinct shapes of these force profile components were predicted by the GF responses to environmental variability directly measured in Experiment 1 and the shape of the expected dynamics, respectively (Fig. 6). This decomposition also revealed, on one hand, that $\sigma$-driven nonspecific learning is present in GF adaptation but absent in MF adaptation, but, on the other, that $\mu$-driven task-specific learning proceeds at remarkably similar rates for GF and MF adaptation (Fig. 7), indicating that previously reported differences in adaptation rate for GF versus MF arose from the failure to take variability-driven GF control into account.

Future work will be needed to pin down the mechanisms by which variability-driven GF control is achieved. It is possible that variability-driven changes in GF control could be achieved without direct estimates of environmental variability. For example, asymmetric trial-to-trial adaptation or asymmetric GF carryover effects could both lead to variability-driven changes in GF control that do not require internal estimates of environmental variability per se. If the adaptive responses that increase GFs following movements with larger than expected LFs are stronger than the responses that decrease GFs following movements with small LFs, higher variability environments, which would have more very large and more very small LFs, would result in trial-to-trial adaptation that is systematically biased toward GF increases. Along similar lines, GFs have been shown to carryover from one trial to the next (Quaney et al., 2003), and if this carryover were larger for above-average than for below-average GFs, higher variability environments might result in carryover that is systematically biased toward GF increases. Preliminary analysis of the current data suggests that GF carryover is small and that trial-to-trial adaptation is largely symmetric, making these asymmetry related hypotheses less likely. But this is yet to be systematically investigated. An attractive hypothesis is that the motor system specifically constructs a running estimate of environmental variability or uncertainty, and directly uses such an estimate in GF control. However, even if this were the case, the mechanisms by which uncertainty is estimated remain to be uncovered.

\section{Previous ideas about safety margins for GF control}

Our results challenge the longstanding idea that, in precision grip tasks, the motor system maintains a constant GF/LF ratio (Johansson and Westling, 1984; Westling and Johansson, 1984), resulting in a safety margin that is a fixed fraction of the expected LF dynamics and is thus insensitive to LF uncertainty. In contrast, our data show that GF is threefold more sensitive to LF variability than to the expected LF, resulting in GF/LF ratios that are readily modulated by environmental uncertainty rather than remaining fixed.

The sensitivity of GF control to environmental variability may have been difficult to appreciate in previous studies due to a focus on static paradigms that are likely to incur substantially smaller uncertainty than paradigms that focus on transient dynamics during rapid motion. For example, the predominant paradigm for studying GF control has involved the maintenance of a single static posture during slow lift-and-hold tasks (Johansson and Westling, 1984, 1987, 1988; Westling and Johansson, 1984; Cole and Johansson, 1993). However, even in these tasks, the role of variability in driving GF control may have been underappreciated. In particular, the finding that the safety margin for GF control scales linearly with the LF in a static hold task (Westling and Johansson, 1984; Cole and Johansson, 1993) may be a variabilitydriven rather than an expected value-driven effect. Although variability was not explicitly manipulated, both the force variability associated with motor output (Jones et al., 2002) and the perceptual variability associated with weight estimation (Holway and Pratt, 1936; Weber, 1978) scale linearly with the load. In fact, Weber's Law, which maintains that perceptual uncertainty is generally proportional to intensity, was originally based on studies of weight perception (Weber, 1978). These observations make it clear that in static tasks, the safety margin for GF control is simultaneously proportional to weight estimate variability, mo- 
tor output variability, and the LF itself. It would be interesting to determine which of these effects dominate.

\section{Previous findings comparing the rates of GF and MF adaptation}

A key finding from Experiment 2 is that GF adaptation is not consistently faster than MF adaptation, as previous work has suggested (Flanagan et al., 2003). We find that the environmentspecific mean-driven response that is present in both MF and GF control adapts at essentially identical rates for MF and GF. The apparent difference between the rates of overall GF and MF adaptation observed when only LF increases were examined has been taken as general evidence for a GF rate advantage and used to support the idea that a forward model for predicting the sensory consequences of environmental dynamics adapts faster than an inverse model (Flanagan et al., 2003). However, the current findings suggest this conclusion to be problematic. Instead, faster adaptation for GF compared with MF is confined to instances in which the LF increases, with the opposite observed when LF decreases. The mechanism here is that variability-driven responses act to increase GF whenever the environment changes, regardless of whether that change would require smaller or larger GFs. Thus, this nonspecific variability-driven adaptive response synergizes with environment-specific mean-driven adaptation when LF increases, but interferes with it when LF decreases, leading to dramatic differences in adaptation rates. However, this dichotomy does not occur with MF adaptation, because MF is insensitive to environmental variability. Our findings also provide explanations for previous results showing that GFs are paradoxically increased following initial exposure to microgravity (Augurelle et al., 2003) and that real-time feedback responses can lead to increased GF following sudden, unexpected increases or decreases in LF (Danion, 2007). These results mirror the current findings, and suggest that variability-driven changes in GF control govern real-time feedback responses as well as feedforward motor adaptation. Moreover, the finding that environment-specific MF and GF adaptation proceeds at essentially identical rates suggests a unified mechanism for the adaptation of internal models for the expected environmental dynamics in the control of manipulatory and grip forces.

\section{Uncertainty-driven control of GFs and muscular cocontraction}

Uncertainty-driven safety margins, like the ones we observed in the control of GF, may explain previous observations about the control of cocontraction of antagonist muscle pairs. Like GF control, antagonist muscle control consists of specific and nonspecific components: reciprocal activation (which drives the exerted MF and is specific to the expected environmental dynamics) and cocontraction (which has no effect on the net MF and is nonspecific to environmental dynamics; Feldman, 1980; Franklin and Wolpert, 2011). Cocontraction increases the mechanical stiffness of the limb in resisting unexpected perturbations, allowing for improved control in uncertain environments (Franklin et al., 2003, 2004, 2008; Milner and Franklin, 2005). While not providing a frank safety margin that prevents errors from unexpected perturbations, the increased ability to reduce errors stemming from unexpected perturbations that is afforded by cocontraction make it like a "soft" safety margin that suppresses such unexpected perturbations, raising the possibility that cocontraction may be controlled in a fashion similar to the mechanism we describe for GF. Indeed, several key features of cocontraction are consistent with uncertainty-driven control. Cocontraction in- creases immediately after detection of an unexpected load (Smeets et al., 1990) and, more generally, while moving in dynamic environments of increased trial-to-trial variability (Takahashi et al., 2001), but only transiently following step changes in dynamics (Milner and Cloutier, 1993; Thoroughman and Shadmehr, 1999; Franklin et al., 2003, 2004).

\section{Impact of neurologic disease on uncertainty-driven GF control}

Increased uncertainty for GF control can also reflect imprecise sensory information about the environment, or imprecise control of motor output. Thus the current results based on the manipulation of environmental variability predict that increased sensory or motor uncertainty will also increase GF safety margins, leading to consistently higher GF. This may explain why both healthy subjects under anesthesia (Westling and Johansson, 1984; Cole and Abbs, 1988; Nowak et al., 2001) and patients with a range of sensorimotor dysfunction exhibit elevated GF safety margins. In particular, elevated GFs have been reported in patients with multiple sclerosis (Iyengar et al., 2009), Parkinson's disease (Fellows et al., 1998; Wenzelburger et al., 2002), carpal tunnel syndrome (Zhang et al., 2011; Afifi et al., 2012), peripheral deafferentation (Nowak et al., 2003), and cerebral stroke (Hermsdörfer et al., 2003; Dubrowski et al., 2005; Anens et al., 2010).

\section{Uncertainty estimation and metacognition}

Estimates of uncertainty — or, inversely, confidence-about the state of the environment related to one's decisions or actions have traditionally been classified as metacognitive abilities which encompass "what we know about what we know" (Metcalfe and Shimamura, 1994; Nelson, 1996; Hacker, 1998). But metacognitive abilities have been considered to be exclusive to humans and primates (Metcalfe, 2008), whereas recent work on decision making in rodents has identified behavioral and neural correlates of confidence (Foote and Crystal, 2007; Kepecs et al., 2008; Terrace and Son, 2009; Kepecs and Mainen, 2012). This should not come as a surprise when considering motor control, given the longstanding hypothesis that the motor system relies on internal estimates of variability and uncertainty for motor planning. It has been widely hypothesized that movements are specifically planned to minimize the task-relevant component of motor variability, possibly in combination with effort (Harris and Wolpert, 1998; Todorov and Jordan, 2002; Izawa and Shadmehr, 2008; Izawa et al., 2008; Braun et al., 2009). Moreover, the strength of on-line movement corrections has been shown to depend both on the certainty associated with sensory information and one's prior beliefs about the environment in a manner consistent with Bayesian integration (Körding and Wolpert, 2004; Körding et al., 2004; Franklin et al., 2012). This integration often occurs without awareness as simple neural computations can provide the required variability estimates (Ma et al., 2006; Kepecs et al., 2008; Kiani and Shadlen, 2009; Kepecs and Mainen, 2012; De Martino et al., 2013). For example, variability estimates like the ones used in the ACEVU model can arise from a simple trial-by-trial update rule, whereby the current estimate of variability can be expressed as a weighted combination of the previous variability estimate and a rectified version of current sensory information (Eqs. 2 and $3)$. Thus uncertainty estimates need not be the exclusive domain of high-level cognitive endeavors; in fact, our work illustrates that they are indeed an integral part of the machinery involved in performing basic motor actions that do not require explicit specification, like controlling grip forces. Moreover, the exquisite sen- 
sitivity of GFs to environmental variability that we demonstrate identifies GF control as a valuable paradigm for probing the nervous system's estimates of uncertainty. Going forward, this establishes a new tool for better understanding the mechanisms underlying the estimation of uncertainty in the nervous system about which little is currently known.

\section{Notes}

Supplemental material for this article is available at http://www.seas. harvard.edu/motorlab/Hadjiosif_and_Smith_safety_margin_paper/ Supplementary_Materials.pdf. Figures and analysis show (1) force profiles in Experiment 1b, (2) the robustness of results under different metrics, (3) individual subject responses, (4) GF responses for EC versus normal FF trials in Experiment 1a, illustrating sensitivity to variability in both cases, (5) premovement GF levels, and (6) an examination of possible trial-to-trial carryover effects for GF. This material has not been peer reviewed.

\section{References}

ACI Committee 318 (2005) Building code requirements for structural concrete (ACI 318-05) and commentary (ACI 318R-05). Farmington Hills, MI: American Concrete Institute.

Afifi M, Santello M, Johnston JA (2012) Effects of carpal tunnel syndrome on adaptation of multi-digit forces to object texture. Clin Neurophysiol 123:2281-2290. CrossRef Medline

Anens E, Kristensen B, Häger-Ross C (2010) Reactive grip force control in persons with cerebellar stroke: effects on ipsilateral and contralateral hand. Exp Brain Res 203:21-30. CrossRef Medline

Augurelle AS, Penta M, White O, Thonnard JL (2003) The effects of a change in gravity on the dynamics of prehension. Exp Brain Res 148:533540. Medline

Braun DA, Aertsen A, Wolpert DM, Mehring C (2009) Learning optimal adaptation strategies in unpredictable motor tasks. J Neurosci 29:64726478. CrossRef Medline

Burge J, Ernst MO, Banks MS (2008) The statistical determinants of adaptation rate in human reaching. J Vis 8(4):20.1-19. CrossRef Medline

Cole KJ, Abbs JH (1988) Grip force adjustments evoked by load force perturbations of a grasped object. J Neurophysiol 60:1513-1522. Medline

Cole KJ, Johansson RS (1993) Friction at the digit-object interface scales the sensorimotor transformation for grip responses to pulling loads. Exp Brain Res 95:523-532. Medline

Danion F (2007) The contribution of non-digital afferent signals to grip force adjustments evoked by brisk unloading of the arm or the held object. Clin Neurophysiol 118:146-154. CrossRef Medline

Danion F, Sarlegna FR (2007) Can the human brain predict the consequences of arm movement corrections when transporting an object? Hints from grip force adjustments. J Neurosci 27:12839-12843. CrossRef Medline

De Martino B, Fleming SM, Garrett N, Dolan RJ (2013) Confidence in value-based choice. Nat Neurosci 16:105-110. CrossRef Medline

Donchin O, Francis JT, Shadmehr R (2003) Quantifying generalization from trial-by-trial behavior of adaptive systems that learn with basis functions: theory and experiments in human motor control. J Neurosci 23: 9032-9045. Medline

Dubrowski A, Roy EA, Black SE, Carnahan H (2005) Unilateral basal ganglia damage causes contralesional force control deficits: a case study. Neuropsychologia 43:1379-1384. CrossRef Medline

Feldman AG (1980) Superposition of motor programs-I. Rhythmic forearm movements in man. Neuroscience 5:81-90. CrossRef Medline

Fellows SJ, Noth J, Schwarz M (1998) Precision grip and Parkinson's disease. Brain 121:1771-1784. CrossRef Medline

Flanagan JR, Wing AM (1993) Modulation of grip force with load force during point-to-point arm movements. Exp Brain Res 95:131-143. Medline

Flanagan JR, Wing AM (1997) The role of internal models in motion planning and control: evidence from grip force adjustments during movements of hand-held loads. J Neurosci 17:1519-1528. Medline

Flanagan JR, Vetter P, Johansson RS, Wolpert DM (2003) Prediction precedes control in motor learning. Curr Biol 13:146-150. CrossRef Medline

Foote AL, Crystal JD (2007) Metacognition in the rat. Curr Biol 17:551-555. CrossRef Medline
Franklin DW, Wolpert DM (2011) Computational mechanisms of sensorimotor control. Neuron 72:425-442. CrossRef Medline

Franklin DW, Osu R, Burdet E, Kawato M, Milner TE (2003) Adaptation to stable and unstable dynamics achieved by combined impedance control and inverse dynamics model. J Neurophysiol 90:3270-3282. CrossRef Medline

Franklin DW, So U, Kawato M, Milner TE (2004) Impedance control balances stability with metabolically costly muscle activation. J Neurophysiol 92:3097-3105. CrossRef Medline

Franklin DW, Burdet E, Tee KP, Osu R, Chew CM, Milner TE, Kawato M (2008) CNS learns stable, accurate, and efficient movements using a simple algorithm. J Neurosci 28:11165-11173. CrossRef Medline

Franklin S, Wolpert DM, Franklin DW (2012) Visuomotor feedback gains upregulate during the learning of novel dynamics. J Neurophysiol 108: 467-478. CrossRef Medline

Fu Q, Zhang W, Santello M (2010) Anticipatory planning and control of grasp positions and forces for dexterous two-digit manipulation. J Neurosci 30:9117-9126. CrossRef Medline

Goldsmith M (1956) Pablo Casals talks of modern music. News and Courier (Charleston, SC) 9C.

Gonzalez Castro LN, Monsen CB, Smith MA (2011) The binding of learning to action in motor adaptation. Plos Comput Biol 7:e1002052. CrossRef Medline

Gonzalez Castro LN, Hadjiosif AM, Hemphill MA, Smith MA (2014) Environmental consistency determines the rate of motor adaptation. Curr Biol 24:1050-1061. CrossRef Medline

Hacker DJ (1998) Definitions and empirical foundations. In: Metacognition in educational theory and practice (Hacker DJ, Dunlosky J, Graesser AC, eds), pp 1-24. New York: Taylor \& Francis.

Harris CM, Wolpert DM (1998) Signal-dependent noise determines motor planning. Nature 394:780-784. CrossRef Medline

Hermsdörfer J, Hagl E, Nowak DA, Marquardt C (2003) Grip force control during object manipulation in cerebral stroke. Clin Neurophysiol 114: 915-929. CrossRef Medline

Holway AH, Pratt CC (1936) The Weber-ratio for intensitive discrimination. Psychol Rev 43:322-340. CrossRef

Iyengar V, Santos MJ, Ko M, Aruin AS (2009) Grip force control in individuals with multiple sclerosis. Neurorehabil Neural Repair 23:855-861. CrossRef Medline

Izawa J, Shadmehr R (2008) On-line processing of uncertain information in visuomotor control. J Neurosci 28:11360-11368. CrossRef Medline

Izawa J, Rane T, Donchin O, Shadmehr R (2008) Motor adaptation as a process of reoptimization. J Neurosci 28:2883-2891. CrossRef Medline

Johansson RS, Westling G (1984) Roles of glabrous skin receptors and sensorimotor memory in automatic control of precision grip when lifting rougher or more slippery objects. Exp Brain Res 56:550-564. Medline

Johansson RS, Westling G (1987) Signals in tactile afferents from the fingers eliciting adaptive motor responses during precision grip. Exp Brain Res 66:141-154. Medline

Johansson RS, Westling G (1988) Programmed and triggered actions to rapid load changes during precision grip. Exp Brain Res 71:72-86. Medline

Joiner WM, Smith MA (2008) Long-term retention explained by a model of short-term learning in the adaptive control of reaching. J Neurophysiol 100:2948-2955. CrossRef Medline

Joiner WM, Ajayi O, Sing GC, Smith MA (2011) Linear hypergeneralization of learned dynamics across movement speeds reveals anisotropic, gainencoding primitives for motor adaptation. J Neurophysiol 105:45-59. CrossRef Medline

Joiner WM, Brayanov JB, Smith MA (2013) The training schedule affects the stability, not the magnitude, of the interlimb transfer of learned dynamics. J Neurophysiol 110:984-998. CrossRef Medline

Jones KE, Hamilton AF, Wolpert DM (2002) Sources of signal-dependent noise during isometric force production. J Neurophysiol 88:1533-1544. Medline

Kawato M (1999) Internal models for motor control and trajectory planning. Curr Opin Neurobiol 9:718-727. CrossRef Medline

Kepecs A, Mainen ZF (2012) A computational framework for the study of confidence in humans and animals. Philos Trans R Soc Lond B Biol Sci 367:1322-1337. CrossRef Medline

Kepecs A, Uchida N, Zariwala HA, Mainen ZF (2008) Neural correlates, 
computation and behavioural impact of decision confidence. Nature 455: 227-231. CrossRef Medline

Kiani R, Shadlen MN (2009) Representation of confidence associated with a decision by neurons in the parietal cortex. Science 324:759-764. CrossRef Medline

Körding KP, Wolpert DM (2004) Bayesian integration in sensorimotor learning. Nature 427:244-247. CrossRef Medline

Körding KP, Ku SP, Wolpert DM (2004) Bayesian integration in force estimation. J Neurophysiol 92:3161-3165. CrossRef Medline

Krakauer JW, Ghilardi MF, Ghez C (1999) Independent learning of internal models for kinematic and dynamic control of reaching. Nat Neurosci 2:1026-1031. CrossRef Medline

Landy MS, Trommershäuser J, Daw ND (2012) Dynamic estimation of task-relevant variance in movement under risk. J Neurosci 32:1270212711. CrossRef Medline

Lee JY, Schweighofer N (2009) Dual adaptation supports a parallel architecture of motor memory. J Neurosci 29:10396-10404. CrossRef Medline

Ma WJ, Beck JM, Latham PE, Pouget A (2006) Bayesian inference with probabilistic population codes. Nat Neurosci 9:1432-1438. CrossRef Medline

Marsaglia G (1965) Ratios of normal variables and ratios of sums of uniform variables. J Am Stat Assoc 60:193-204. CrossRef

Mawase F, Karniel A (2012) Adaptation to sequence force perturbation during vertical and horizontal reaching movement-averaging the past or predicting the future? Front Syst Neurosci 6:60. CrossRef Medline

Metcalfe J (2008) Evolution of metacognition. In: Handbook of metamemory and memory (Dunlosky J, Bjork R, eds), pp 29-46. New York: Psychology.

Metcalfe J, Shimamura AP (1994) Metacognition: knowing about knowing. Cambridge, MA: MIT.

Milner TE, Cloutier C (1993) Compensation for mechanically unstable loading in voluntary wrist movement. Exp Brain Res 94:522-532. Medline

Milner TE, Franklin DW (2005) Impedance control and internal model use during the initial stage of adaptation to novel dynamics in humans. J Physiol 567:651-664. CrossRef Medline

NASA Technical Standards Program (2008) Structural design and test factors of safety for spaceflight hardware. Washington, DC: National Aeuronautics and Space Administration.

Nelson TO (1996) Consciousness and metacognition. Am Psychol 51:102116. CrossRef

Nowak DA, Hermsdörfer J, Glasauer S, Philipp J, Meyer L, Mai N (2001) The effects of digital anaesthesia on predictive grip force adjustments during vertical movements of a grasped object. Eur J Neurosci 14:756762. CrossRef Medline

Nowak DA, Glasauer S, Hermsdörfer J (2003) Grip force efficiency in longterm deprivation of somatosensory feedback. Neuroreport 14:1803-1807. CrossRef Medline

Pekny SE, Criscimagna-Hemminger SE, Shadmehr R (2011) Protection and expression of human motor memories. J Neurosci 31:13829-13839. CrossRef Medline

Quaney BM, Rotella DL, Peterson C, Cole KJ (2003) Sensorimotor memory for fingertip forces: evidence for a task-independent motor memory. J Neurosci 23:1981-1986. Medline

Saijo N, Gomi H (2012) Effect of visuomotor-map uncertainty on visuomotor adaptation. J Neurophysiol 107:1576-1585. CrossRef Medline

Scheidt RA, Reinkensmeyer DJ, Conditt MA, Rymer WZ, Mussa-Ivaldi FA (2000) Persistence of motor adaptation during constrained, multi-joint, arm movements. J Neurophysiol 84:853-862. Medline

Scheidt RA, Dingwell JB, Mussa-Ivaldi FA (2001) Learning to move amid uncertainty. J Neurophysiol 86:971-985. Medline
Shadmehr R, Mussa-Ivaldi FA (1994) Adaptive representation of dynamics during learning of a motor task. J Neurosci 14:3208-3224. Medline

Sing GC, Smith MA (2010) Reduction in learning rates associated with anterograde interference results from interactions between different timescales in motor adaptation. Plos Comput Biol 6:pii:e1000893. CrossRef Medline

Sing GC, Joiner WM, Nanayakkara T, Brayanov JB, Smith MA (2009) Primitives for motor adaptation reflect correlated neural tuning to position and velocity. Neuron 64:575-589. CrossRef Medline

Sing GC, Orozco SP, Smith MA (2013) Limb motion dictates how motor learning arises from arbitrary environmental dynamics. J Neurophysiol 109:2466-2482. CrossRef Medline

Smeets JB, Erkelens CJ, Denier van der Gon JJ (1990) Adjustments of fast goal-directed movements in response to an unexpected inertial load. Exp Brain Res 81:303-312. CrossRef Medline

Smith MA, Shadmehr R (2005) Intact ability to learn internal models of arm dynamics in Huntington's disease but not cerebellar degeneration. J Neurophysiol 93:2809-2821. CrossRef Medline

Smith MA, Ghazizadeh A, Shadmehr R (2006) Interacting adaptive processes with different timescales underlie short-term motor learning. PLoS Biol 4:e179. CrossRef Medline

Takahashi CD, Scheidt RA, Reinkensmeyer DJ (2001) Impedance control and internal model formation when reaching in a randomly varying dynamical environment. J Neurophysiol 86:1047-1051. Medline

Terrace HS, Son LK (2009) Comparative metacognition. Curr Opin Neurobiol 19:67-74. CrossRef Medline

Thoroughman KA, Shadmehr R (1999) Electromyographic correlates of learning an internal model of reaching movements. J Neurosci 19:85738588. Medline

Thoroughman KA, Shadmehr R (2000) Learning of action through adaptive combination of motor primitives. Nature 407:742-747. CrossRef Medline

Todorov E, Jordan MI (2002) Optimal feedback control as a theory of motor coordination. Nat Neurosci 5:1226-1235. CrossRef Medline

Wasserman L (2004) All of statistics: a concise course in statistical inference. New York: Springer.

Weber EH (1978) The sense of touch. London: Academic.

Wenzelburger R, Zhang BR, Poepping M, Schrader B, Müller D, Kopper F, Fietzek U, Mehdorn HM, Deuschl G, Krack P (2002) Dyskinesias and grip control in Parkinson's disease are normalized by chronic stimulation of the subthalamic nucleus. Ann Neurol 52:240-243. CrossRef Medline

Westling G, Johansson RS (1984) Factors influencing the force control during precision grip. Exp Brain Res 53:277-284. Medline

Wolpert DM, Kawato M (1998) Multiple paired forward and inverse models for motor control. Neural Netw 11:1317-1329. CrossRef Medline

Wolpert DM, Miall RC, Kawato M (1998) Internal models in the cerebellum. Trends Cogn Sci 2:338-347. CrossRef Medline

Wu HG, Miyamoto YR, Gonzalez Castro LN, Ölveczky BP, Smith MA (2014) Temporal structure of motor variability is dynamically regulated and predicts motor learning ability. Nat Neurosci 17:312-321. CrossRef Medline

Yokoi A, Hirashima M, Nozaki D (2011) Gain field encoding of the kinematics of both arms in the internal model enables flexible bimanual action. J Neurosci 31:17058-17068. CrossRef Medline

Yousif N, Diedrichsen J (2012) Structural learning in feedforward and feedback control. J Neurophysiol 108:2373-2382. CrossRef Medline

Zhang W, Johnston JA, Ross MA, Smith AA, Coakley BJ, Gleason EA, Dueck AC, Santello M (2011) Effects of carpal tunnel syndrome on adaptation of multi-digit forces to object weight for whole-hand manipulation. PloS One 6:e27715. CrossRef Medline 